Supporting Information

\title{
Phototropin-Inspired Chemoselective Synthesis of Unsymmetrical Disulfides: Aerobic Oxidative Heterocoupling of Thiols using Flavin Photocatalysis
}

Marina Oka, Daichi Katsube, Takeshi Tsuji, and Hiroki Iida*

Department of Chemistry, Graduate School of Natural Science and Technology, Shimane University, 1060 Nishikawatsu, Matsue, 690-8504, Japan

E-mail address: iida@riko.shimane-u.ac.jp

1. General S2

2. Materials S2

3. Experimental Procedures $\quad \mathrm{S} 2$

4. 'H and ${ }^{13} \mathrm{C}\left\{{ }^{\prime} \mathrm{H}\right\}$ NMR Spectra of Novel Compounds $\quad \mathrm{S} 11$

5. 'H NMR Spectra of Known Compounds S20

6. References $\quad$ S25 


\section{General}

The IR spectra were recorded on a JASCO FT/IR-660plus spectrophotometer (JASCO, Tokyo, Japan). The NMR spectra were measured using JEOL JNM-L400 and JNM ECX-500 spectrometers (JEOL, Akishima, Japan) operating at 400 and $500 \mathrm{MHz}$, respectively, for ${ }^{~} \mathrm{H}$ and 100 and $126 \mathrm{MHz}$, respectively, for ${ }^{13} \mathrm{C}$ using tetramethylsilane (TMS) or a solvent residual peak as the internal standard. The electrospray ionization mass (ESI-MS) spectra were recorded on a Bruker microTOFII-SHIY3 mass spectrometer (Bruker, Billerica, MA) using the positive or negative mode ESI-TOF method for acetonitrile solutions and sodium formate as the reference. The GC measurements were performed on a Shimadzu GC-2014 gas chromatograph (Shimadzu, Kyoto, Japan) equipped with a flame ionization detector (FID) using a Supelco Equity-5 (30 m x $0.25 \mathrm{~mm})$ column.

\section{Materials}

All starting materials were purchased from Aldrich (Milwaukee, WI), Wako Pure Chemical Industries (Osaka, Japan), Nacalai tesque (Kyoto, Japan), and Tokyo Kasei (TCI, Tokyo, Japan) and were used as received. Riboflavin tetraacetate $(\mathbf{F l})^{s !}$ and its reduced form $\left(\mathbf{F l}_{\mathrm{rad}}\right)^{\text {s2 }}$ were synthesized according to the previously reported methods.

\section{Experimental Procedures}

Typical procedure for homocoupling of $\mathbf{1 b}$. A mixture of 4-methoxybenzenethiol (1b, $140 \mathrm{mg}, 1.00 \mathrm{mmol}), \mathbf{F l}(27.2 \mathrm{mg}, 0.050 \mathrm{mmol}), \mathrm{MeOH}(10 \mathrm{~mL}), \mathrm{H}_{2} \mathrm{O}(10 \mathrm{~mL})$, and pyridine $\left(100 \mu \mathrm{L}\right.$ ) was stirred and irradiated (white LED, $11 \mathrm{~W}$ ) at $25{ }^{\circ} \mathrm{C}$ for $2 \mathrm{~h}$ under $\mathrm{O}_{2}$. The yield of the desired disulfide $\mathbf{2 b b}$ was determined by GC measurement. These results are summarized in Scheme 2. 
Typical procedure for synthesis of 2ac. A mixture of 2-pyridylthiol (1a, $111 \mathrm{mg}, 1.00$ mmol), tert-butylthiol (1c, $108 \mathrm{mg}, 1.20 \mathrm{mmol})$, Fl (27.2 mg, $0.050 \mathrm{mmol}), \mathrm{MeOH}(10$ $\mathrm{mL})$, and $\mathrm{H}_{2} \mathrm{O}(10 \mathrm{~mL})$ was stirred and irradiated (white LED, $11 \mathrm{~W}$ ) at $25^{\circ} \mathrm{C}$ for $8 \mathrm{~h}$ under $\mathrm{O}_{2}$. After the solvent was removed by evaporation, the residue was purified by column chromatography $\left(\mathrm{SiO}_{2}\right.$, hexane/ethyl acetate $=100 / 0$ to $\left.4 / 1, \mathrm{v} / \mathrm{v}\right)$ to give $2 \mathbf{a c}$ (155 mg, 78\%) as a colorless oil. These results are summarized in Scheme 3.

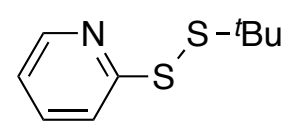

Spectroscopic data of 2-(tert-butyldisulfanyl)pyridine (2ac): Column chromatography $\left(\mathrm{SiO}_{2}\right.$, hexane/ethyl acetate $=100 / 0$ to $\left.4 / 1, \mathrm{v} / \mathrm{v}\right)$ afforded the desired product $(155 \mathrm{mg}$, $78 \%)$ as a colorless oil. ' $\mathrm{H}$ NMR $\left(500 \mathrm{MHz}, \mathrm{CDCl}_{3}, 25^{\circ} \mathrm{C}, \delta\right): 8.44-8.40(\mathrm{~m}, 1 \mathrm{H}), 7.79$ $(\mathrm{d}, J=8.0 \mathrm{~Hz}, 1 \mathrm{H}), 7.65-7.55(\mathrm{~m}, 1 \mathrm{H}), 7.15-7.10(\mathrm{~m}, 1 \mathrm{H}), 1.34(\mathrm{~s}, 9 \mathrm{H}) .{ }^{1} \mathrm{C}\left\{{ }^{1} \mathrm{H}\right\} \mathrm{NMR}$ (126 MHz, $\left.\mathrm{CDCl}_{3}, 25^{\circ} \mathrm{C}, \delta\right): 161.8,149.3,136.9,120.5,119.7,49.4,29.9$.<smiles>CC(C)CSSc1ccncc1</smiles>

Spectroscopic data of 4-(tert-butyldisulfanyl)pyridine (2dc): ${ }^{\text {s3 }}$ Column chromatography $\left(\mathrm{SiO}_{2}\right.$, chloroform/hexane=3/2 to $\left.100 / 0, \mathrm{v} / \mathrm{v}\right)$ afforded the desired product $(151 \mathrm{mg}$, $76 \%)$ as a colorless oil. ' $\mathrm{H}$ NMR $\left(500 \mathrm{MHz} \mathrm{CDCl}_{3}, 25^{\circ} \mathrm{C}, \delta\right): 8.45(\mathrm{~d}, J=6.0 \mathrm{~Hz}, 2 \mathrm{H})$, $7.48(\mathrm{~d}, J=6.0 \mathrm{~Hz}, 2 \mathrm{H}), 1.33(\mathrm{~s}, 9 \mathrm{H}) .{ }^{13} \mathrm{C}\left\{{ }^{\prime} \mathrm{H}\right\} \mathrm{NMR}\left(126 \mathrm{MHz}, \mathrm{CDCl}_{3}, 25^{\circ} \mathrm{C}, \delta\right): 150.3$, $149.4,120.2,49.9,29.9$.<smiles>CC(C)(CCO)SSc1ccccn1</smiles>

Spectroscopic data of 3-methyl-3-(pyridin-2-yldisulfanyl)butan-1-ol (2ae): Column chromatography $\left(\mathrm{SiO}_{2}\right.$, hexane/ethyl acetate $=100 / 0$ to $\left.1 / 1, \mathrm{v} / \mathrm{v}\right)$ afforded the desired product (201 mg, 87\%) as a colorless oil. IR (neat, $\left.\mathrm{cm}^{-1}\right)$ 3354, 3048, 1576, 1560, 1447 , 1417, 762, 718. 'H NMR (500 MHz, $\left.\mathrm{CDCl}_{3}, 25^{\circ} \mathrm{C}, \delta\right): 8.45-8.40(\mathrm{~m}, 1 \mathrm{H}), 7.69$ (d, $J=$ $7.5 \mathrm{~Hz}, 1 \mathrm{H}), 7.61(\mathrm{ddd}, J=7.8,7.8,2.0 \mathrm{~Hz}, 1 \mathrm{H}), 7.08(\mathrm{ddd}, J=7.3,4.5,1.1 \mathrm{~Hz}, 1 \mathrm{H})$, $3.81(\mathrm{t}, J=6.8 \mathrm{~Hz}, 2 \mathrm{H}), 2.59(\mathrm{br} \mathrm{s}, 1 \mathrm{H}), 1.90(\mathrm{t}, J=6.5 \mathrm{~Hz}, 2 \mathrm{H}), 1.36(\mathrm{~s}, 6 \mathrm{H}) .{ }^{\mathrm{B}} \mathrm{C}\left\{{ }^{1} \mathrm{H}\right\}$ 
NMR (126 MHz, $\left.\mathrm{CDCl}_{3}, 25^{\circ} \mathrm{C}, \delta\right): 160.8,149.4,137.0,120.8,120.4,59.7,51.1,43.3$, 28.1. HRMS (ESI-TOF) m/z: [M+H] ${ }^{+}$calcd for $\mathrm{C}_{11} \mathrm{H}_{15} \mathrm{NOS}_{2}, 230.0668$; found, 230.0665 .<smiles>CCSSc1ncccn1</smiles>

Spectroscopic data of 2-(tert-butyldisulfanyl)pyrimidine $\mathbf{( 2 f c ) : : ^ { : s 3 }}$ Column chromatography $\left(\mathrm{SiO}_{2}\right.$, dichloromethane/methanol=100/0 to $\left.10 / 1, \mathrm{v} / \mathrm{v}\right)$ afforded the desired product (160 mg, 80\%) as a colorless oil. ' $\mathrm{H}$ NMR $\left(500 \mathrm{MHz}, \mathrm{CDCl}_{3}, 25^{\circ} \mathrm{C}, \delta\right)$ : $8.61(\mathrm{~d}, J=4.5 \mathrm{~Hz}, 2 \mathrm{H}), 7.08(\mathrm{t}, J=4.8 \mathrm{~Hz}, 1 \mathrm{H}), 1.37(\mathrm{~s}, 9 \mathrm{H}) .{ }^{\mathrm{r}} \mathrm{C}\left\{{ }^{\prime} \mathrm{H}\right\} \mathrm{NMR}(126 \mathrm{MHz}$, $\left.\mathrm{CDCl}_{3}, 25^{\circ} \mathrm{C}, \delta\right): 172.6,158.0,118.2,49.4,30.1$.<smiles>CC(C)CSSc1nc2ccccc2s1</smiles>

Spectroscopic data of 2-(tert-butyldisulfanyl)benzothiazole (2gc): ${ }^{\text {s: }}$ Column chromatography $\left(\mathrm{SiO}_{2}\right.$, hexane) afforded the desired product $(238 \mathrm{mg}, 93 \%)$ as a white solid. 'H NMR (500 MHz, $\left.\mathrm{CDCl}_{3}, 25^{\circ} \mathrm{C}, \delta\right): 7.85(\mathrm{~d}, J=8.0 \mathrm{~Hz}, 1 \mathrm{H}), 7.80-7.76(\mathrm{~m}, 1 \mathrm{H})$, 7.45-7.39 (m, 1H), 7.34-7.29 (m, 1H), $1.43(\mathrm{~s}, 9 \mathrm{H}) .{ }^{\mathrm{i}} \mathrm{C}\left\{{ }^{\prime} \mathrm{H}\right\} \mathrm{NMR}\left(126 \mathrm{MHz}, \mathrm{CDCl}_{3}\right.$, $\left.25{ }^{\circ} \mathrm{C}, \delta\right): 174.5,155.0,135.9,126.3,124.6,122.2,121.2,50.4,29.9$.<smiles>Cn1nnnc1SSCBr</smiles>

Spectroscopic data of 5-(tert-butyldisulfanyl)-1-methyl-tetrazole (2hc): ${ }^{\text {ss }}$ Column chromatography $\left(\mathrm{SiO}_{2}\right.$, hexane/ethyl acetate $=100 / 0$ to $\left.1 / 1, \mathrm{v} / \mathrm{v}\right)$ afforded the desired product (169 mg, 82\%) as a colorless oil. ' $\mathrm{H}$ NMR (500 $\mathrm{MHz}, \mathrm{CDCl}_{3}, 25^{\circ} \mathrm{C}, \delta$ ): 4.13 (s, $3 \mathrm{H}), 1.37$ (s, 9H). ${ }^{13} \mathrm{C}\left\{{ }^{\prime} \mathrm{H}\right\} \mathrm{NMR}\left(126 \mathrm{MHz}, \mathrm{CDCl}_{3}, 25^{\circ} \mathrm{C}, \delta\right): 153.6,50.6,34.7,29.7$.<smiles>CC(C)(CCO)SSc1ccccc1</smiles>

Spectroscopic data of 3-methyl-3-(phenyldisulfanyl)butan-1-ol (2ie): Column chromatography $\left(\mathrm{SiO}_{2}\right.$, hexane/ethyl acetate $=100 / 0$ to $\left.7 / 1, \mathrm{v} / \mathrm{v}\right)$ afforded the desired product (201 mg, 88\%) as a colorless oil. IR (neat, $\left.\mathrm{cm}^{-1}\right)$ 3347, 3058, 2959, 1578, 1475, 
1438, 739, 688. 'H NMR (500 MHz, $\left.\mathrm{CDCl}_{3}, 25^{\circ} \mathrm{C}, \delta\right)$ : 7.60-7.54 (m, 2H), $7.30(\mathrm{t}, J=$ $7.8 \mathrm{~Hz}, 2 \mathrm{H}), 7.20(\mathrm{t}, J=7.3 \mathrm{~Hz}, 1 \mathrm{H}), 3.69(\mathrm{t}, J=7.0 \mathrm{~Hz}, 2 \mathrm{H}), 1.86(\mathrm{t}, J=7.0 \mathrm{~Hz}, 2 \mathrm{H})$, $1.30(\mathrm{~s}, 6 \mathrm{H}) .{ }^{13} \mathrm{C}\left\{{ }^{\prime} \mathrm{H}\right\} \mathrm{NMR}\left(126 \mathrm{MHz}, \mathrm{CDCl}_{3}, 25^{\circ} \mathrm{C}, \delta\right): 138.6,129.0,127.7,126.8$, 59.9, 51.3, 43.6, 28.3. Anal. Calcd for $\mathrm{C}_{11} \mathrm{H}_{10} \mathrm{OS}$ : C, 57.85; H, 7.06. Found: C, 57.67; H, 7.22 .<smiles>CC(C)SSc1ccc(Cl)cc1</smiles>

Spectroscopic data of 4-(tert-butyldisulfanyl)-1-chlorobenzene (2je): ${ }^{\text {s3 }}$ Column chromatography $\left(\mathrm{SiO}_{2}\right.$, hexane/ethyl acetate $=100 / 0$ to $\left.4 / 1, \mathrm{v} / \mathrm{v}\right)$ afforded the desired product (197 mg, 85\%) as a colorless oil. ${ }^{\mathrm{H}} \mathrm{NMR}\left(500 \mathrm{MHz}, \mathrm{CDCl}_{3}, 25{ }^{\circ} \mathrm{C}, \delta\right): 7.48(\mathrm{~d}$, $J=9.0 \mathrm{~Hz}, 2 \mathrm{H}), 7.25(\mathrm{~d}, J=9.0 \mathrm{~Hz}, 2 \mathrm{H}), 1.29(\mathrm{~s}, 9 \mathrm{H}) .{ }^{\mathrm{r}} \mathrm{C}\left\{{ }^{\prime} \mathrm{H}\right\} \mathrm{NMR}\left(126 \mathrm{MHz}, \mathrm{CDCl}_{3}\right.$, $\left.25^{\circ} \mathrm{C}, \delta\right): 137.6,132.2,129.0,128.2,49.6,30.0$.<smiles>COc1ccc(SSCC(C)(C)C)cc1</smiles>

Spectroscopic data of 4-(tert-butyldisulfanyl)-1-methoxybenzene (2bc): ${ }^{\text {ss }}$ Column chromatography $\left(\mathrm{SiO}_{2}\right.$, hexane) afforded the desired product $(148 \mathrm{mg}, 65 \%)$ as a colorless oil. 'H NMR (500 MHz, $\left.\mathrm{CDCl}_{3}, 25^{\circ} \mathrm{C}, \delta\right): 7.48$ (d, $\left.J=8.5 \mathrm{~Hz}, 2 \mathrm{H}\right), 6.83(\mathrm{~d}, J$ $=9.0 \mathrm{~Hz}, 2 \mathrm{H}), 3.79(\mathrm{~s}, 3 \mathrm{H}), 1.29(\mathrm{~s}, 9 \mathrm{H}) .{ }^{\mathrm{i}} \mathrm{C}\left\{{ }^{\prime} \mathrm{H}\right\} \mathrm{NMR}\left(126 \mathrm{MHz}, \mathrm{CDCl}_{3}, 25^{\circ} \mathrm{C}, \delta\right)$ : $159.1,130.4,129.9,114.6,55.5,49.1,30.1$.

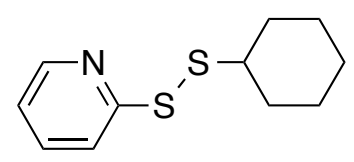

Spectroscopic data of 2-(cyclohexyldisulfanyl)pyridine (2ak): Column chromatography $\left(\mathrm{SiO}_{2}\right.$, hexane/ethyl acetate $=100 / 0$ to $\left.2 / 1, \mathrm{v} / \mathrm{v}\right)$ afforded the desired product $(155 \mathrm{mg}$, $69 \%$ ) as a colorless oil. IR (neat, $\mathrm{cm}^{-1}$ ) 2928, 1573, 1560, 1445, 1416, 760, 717. 'H NMR $\left(500 \mathrm{MHz}, \mathrm{CDCl}_{3}, 25^{\circ} \mathrm{C}, \delta\right): 8.48-8.38(\mathrm{~m}, 1 \mathrm{H}), 7.77(\mathrm{~d}, J=8.0 \mathrm{~Hz}, 1 \mathrm{H}), 7.66-7.59(\mathrm{~m}$, $1 \mathrm{H}), 7.09-7.01(\mathrm{~m}, 1 \mathrm{H}), 2.92-2.79(\mathrm{~m}, 1 \mathrm{H}), 2.06-1.98(\mathrm{~m}, 2 \mathrm{H}), 1.83-1.69(\mathrm{~m}, 2 \mathrm{H})$, 1.66-1.50 (m, 1H), 1.49-1.15 (m, 5H). ${ }^{3} \mathrm{C}\left\{{ }^{1} \mathrm{H}\right\}$ NMR (126 MHz, $\left.\mathrm{CDCl}_{3}, 25^{\circ} \mathrm{C}, \delta\right): 161.6$, 
149.4, 137.0, 120.4 119.5, 50.1, 32.8, 26.2, 25.6. HRMS (ESI-TOF) m/z: $[\mathrm{M}+\mathrm{H}]^{+}$calcd for $\mathrm{C}_{12} \mathrm{H}_{10} \mathrm{NS}_{2}, 226.0719$; found, 226.0717.

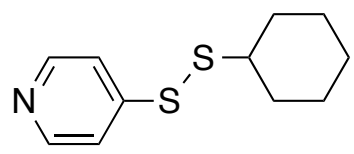

Spectroscopic data of 4-(cyclohexyldisulfanyl)pyridine (2dk): ${ }^{\text {s4 }}$ Column chromatography (alumina, hexane/chloroform $=2 / 3$ to $0 / 100, \mathrm{v} / \mathrm{v}$ ) afforded the desired product (196 mg, 87\%) as a colorless oil. ' $\mathrm{H}$ NMR (500 $\mathrm{MHz}, \mathrm{CDCl}_{3}, 25{ }^{\circ} \mathrm{C}, \delta$ ): 8.48-8.42 (m, 2H), 7.48-7.43 (m, 2H), 2.86-2.77 (m, 1H), 2.04-1.97 (m, 2H), 1.82-1.74 $(\mathrm{m}, 2 \mathrm{H}), 1.65-1.56(\mathrm{~m}, 1 \mathrm{H}), 1.45-1.16(\mathrm{~m}, 5 \mathrm{H}) .{ }^{\mathrm{a}} \mathrm{C}\left\{{ }^{\prime} \mathrm{H}\right\} \mathrm{NMR}\left(126 \mathrm{MHz}, \mathrm{CDCl}_{3}, 25{ }^{\circ} \mathrm{C}\right.$, $\delta): 150.2,149.5,120.0,50.3,32.8,26.1,25.5$.

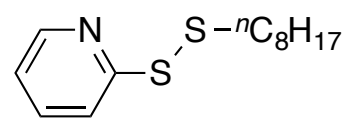

Spectroscopic data of 2-(octyldisulfanyl)pyridine (2al): ${ }^{\text {ss }}$ Column chromatography (alumina, hexane/ethyl acetate $=100 / 0$ to $3 / 1, \mathrm{v} / \mathrm{v}$ ) afforded the desired product $(178 \mathrm{mg}$, $70 \%)$ as a colorless oil. ' $\mathrm{H}$ NMR $\left(500 \mathrm{MHz}, \mathrm{CDCl}_{3}, 25^{\circ} \mathrm{C}, \delta\right): 8.48-8.43(\mathrm{~m}, 1 \mathrm{H}), 7.73$ $(\mathrm{d}, J=8.0 \mathrm{~Hz}, 1 \mathrm{H}), 7.67-7.60(\mathrm{~m}, 1 \mathrm{H}), 7.10-7.04(\mathrm{~m}, 1 \mathrm{H}), 2.79(\mathrm{t}, J=7.5 \mathrm{~Hz}, 2 \mathrm{H})$, 1.76-1.63 (m, 2H), 1.43-1.33 (m, 2H), 1.33-1.19 (m, 8H), $0.87(\mathrm{t}, J=6.8 \mathrm{~Hz}, 3 \mathrm{H})$. ${ }^{13} \mathrm{C}\left\{{ }^{\prime} \mathrm{H}\right\} \mathrm{NMR}\left(126 \mathrm{MHz}, \mathrm{CDCl}_{3}, 25^{\circ} \mathrm{C}, \delta\right): 160.9,149.7,137.0,120.6,119.6,39.2,31.9$, $29.3,29.1,28.6,22.7,14.2$.

$$
\mathrm{C}_{8} \mathrm{H}_{17}-\mathrm{S}^{\prime} \mathrm{S}^{-\mathrm{t}_{\mathrm{Bu}}}
$$

Spectroscopic data of 1-(tert-butyldisulfanyl)octane (2lc): Column chromatography $\left(\mathrm{SiO}_{2}\right.$, hexane/ethyl acetate $=100 / 0$ to $\left.1 / 1, \mathrm{v} / \mathrm{v}\right)$ afforded the desired product $(158 \mathrm{mg}$, $67 \%$ ) as a colorless oil. IR (neat, $\mathrm{cm}^{-1}$ ) 2957, 2925, 2855, 1456, 1362. 'H NMR (500 $\left.\mathrm{MHz}, \mathrm{CDCl}_{3}, 25^{\circ} \mathrm{C}, \delta\right): 2.70(\mathrm{t}, J=7.5 \mathrm{~Hz}, 2 \mathrm{H}), 1.68-1.60(\mathrm{~m}, 2 \mathrm{H}), 1.42-1.20(\mathrm{~m}, 19 \mathrm{H})$, $0.88(\mathrm{t}, J=7.0 \mathrm{~Hz}, 3 \mathrm{H}) .{ }^{1} \mathrm{C}\left\{{ }^{\prime} \mathrm{H}\right\} \mathrm{NMR}\left(126 \mathrm{MHz} \mathrm{CDCl}_{3}, 25{ }^{\circ} \mathrm{C}, \delta\right): 47.8,41.2,32.0$, 30.1 , 29.5, 29.3, 28.7, 22.8, 14.2. Anal. Calcd for $\mathrm{C}_{12} \mathrm{H}_{26} \mathrm{~S}_{2}$ : C, 61.47; H, 11.18. Found: C, $61.53 ; \mathrm{H}, 11.02$. 


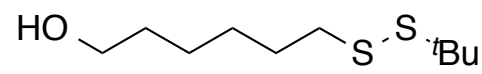

Spectroscopic data of 6-(tert-butyldisulfanyl)hexan-1-ol $\quad$ (2mc): Column chromatography $\left(\mathrm{SiO}_{2}\right.$, hexane/ethyl acetate $=100 / 0$ to $\left.1 / 1, \mathrm{v} / \mathrm{v}\right)$ afforded the desired product (162 $\mathrm{mg}, 70 \%$ ) as a colorless oil. IR (neat, $\left.\mathrm{cm}^{-1}\right)$ 3334, 2929, 726. 'H NMR (500 $\left.\mathrm{MHz}, \mathrm{CDCl}_{3}, 25^{\circ} \mathrm{C}, \delta\right): 3.64(\mathrm{t}, J=6.5 \mathrm{~Hz}, 2 \mathrm{H}), 2.71(\mathrm{t}, J=7.3 \mathrm{~Hz}, 2 \mathrm{H}), 1.72-1.63(\mathrm{~m}$, $2 \mathrm{H}), 1.62-1.54(\mathrm{~m}, 2 \mathrm{H}), 1.48(\mathrm{br} \mathrm{s}, 1 \mathrm{H}), 1.46-1.34(\mathrm{~m}, 4 \mathrm{H}), 1.33(\mathrm{~s}, 9 \mathrm{H}) .{ }^{\mathrm{B}} \mathrm{C}\left\{{ }^{\prime} \mathrm{H}\right\} \mathrm{NMR}$ (126 MHz, $\mathrm{CDCl}_{3}, 25{ }^{\circ} \mathrm{C}, \delta$ ): 63.0, 47.8, 40.9, 32.7, 30.1, 29.4, 28.4, 25.5. Anal. Calcd for $\mathrm{C}_{10} \mathrm{H}_{22} \mathrm{OS}_{2}$ : C, 54.01; H, 9.97. Found: C, 53.94; H, 10.09.

$\overbrace{\mathrm{HO}^{-}} \mathrm{S}^{{ }_{\mathrm{t}} \mathrm{Bu}}$

Spectroscopic data of 3-(tert-butyldisulfanyl)propan-1-ol (2nc): Column chromatography $\left(\mathrm{SiO}_{2}\right.$, hexane/ethyl acetate $=100 / 0$ to $\left.3 / 1, \mathrm{v} / \mathrm{v}\right)$ afforded the desired product $(119 \mathrm{mg}, 66 \%)$ as a colorless oil. IR (neat, $\left.\mathrm{cm}^{-1}\right)$ 3334, 2959. 'H NMR (500 MHz, $\left.\mathrm{CDCl}_{3}, 25{ }^{\circ} \mathrm{C}, \delta\right): 3.75(\mathrm{t}, J=6.3 \mathrm{~Hz}, 2 \mathrm{H}), 2.82(\mathrm{t}, J=7.0 \mathrm{~Hz}, 2 \mathrm{H}), 1.93(\mathrm{tt}, J=7.0,6.3$ $\mathrm{Hz}, 2 \mathrm{H}), 1.63$ (br s, $1 \mathrm{H}), 1.34(\mathrm{~s}, 9 \mathrm{H}) .{ }^{\mathrm{a}} \mathrm{C}\left\{{ }^{\prime} \mathrm{H}\right\} \mathrm{NMR}\left(126 \mathrm{MHz}, \mathrm{CDCl}_{3}, 25{ }^{\circ} \mathrm{C}, \delta\right.$ ): 61.2, 48.0, 37.2, 32.1, 30.1. Anal. Calcd for $\mathrm{C}_{7} \mathrm{H}_{10} \mathrm{OS}_{2}$ : C, 46.63; H, 8.94. Found: $\mathrm{C}$, 46.38; $\mathrm{H}$, 9.08 .<smiles>COC(=O)CCSSC(C)(C)CCO</smiles>

Spectroscopic data of methyl 3-((4-hydroxy-2-methylbutan-2-yl)disulfanyl)propanoate (2oe): Column chromatography $\left(\mathrm{SiO}_{2}\right.$, hexane/ethyl acetate $=100 / 0$ to $\left.3 / 1, \mathrm{v} / \mathrm{v}\right)$ afforded the desired product (174 $\mathrm{mg}, 76 \%$ ) as a colorless oil. IR (neat, $\left.\mathrm{cm}^{-1}\right)$ 3422, 2956, 1738. 'H NMR (500 MHz, $\left.\mathrm{CDCl}_{3}, 25^{\circ} \mathrm{C}, \delta\right): 3.78(\mathrm{t}, J=7.0 \mathrm{~Hz}, 2 \mathrm{H}), 3.71(\mathrm{~s}, 3 \mathrm{H}), 2.94(\mathrm{t}, J=$ $7.3 \mathrm{~Hz}, 2 \mathrm{H}), 2.72$ (t, $J=7.3 \mathrm{~Hz}, 2 \mathrm{H}), 1.90(\mathrm{t}, J=7.0 \mathrm{~Hz}, 2 \mathrm{H}), 1.75$ (br s, 1H), 1.34 (s, $6 \mathrm{H}) .{ }^{{ }^{\prime} \mathrm{C}}\left\{{ }^{\prime} \mathrm{H}\right\} \mathrm{NMR}\left(126 \mathrm{MHz}, \mathrm{CDCl}_{3}, 25^{\circ} \mathrm{C}, \delta\right): 172.4,59.9,52.0,50.0,43.6,34.8,34.2$, 28.3. Anal. Calcd for $\mathrm{C}_{9} \mathrm{H}_{18} \mathrm{O}_{3} \mathrm{~S}_{2}:$ C, 45.35; H, 7.61. Found: C, 45.16; H, 7.84.<smiles>CC(C)(CCO)SS[PbH2]</smiles>

Spectroscopic data of 3-(octyldisulfanyl)-3-methylbutan-1-ol (2le): Column chromatography $\left(\mathrm{SiO}_{2}\right.$, hexane/ethyl acetate $=100 / 0$ to $\left.1 / 1, \mathrm{v} / \mathrm{v}\right)$ afforded the desired 
product (169 $\mathrm{mg}, 65 \%)$ as a colorless oil. IR (neat, $\left.\mathrm{cm}^{-1}\right)$ 3335, 2925. 'H NMR (500 MHz, $\left.\mathrm{CDCl}_{3}, 25^{\circ} \mathrm{C}, \delta\right): 3.79(\mathrm{t}, J=7.0 \mathrm{~Hz}, 2 \mathrm{H}), 2.71(\mathrm{t}, J=7.5 \mathrm{~Hz}, 2 \mathrm{H}), 1.90(\mathrm{t}, J=6.8 \mathrm{~Hz}$, $2 \mathrm{H}), 1.68-1.57(\mathrm{~m}, 3 \mathrm{H}), 1.40-1.21(\mathrm{~m}, 16 \mathrm{H}), 0.88(\mathrm{t}, J=6.8 \mathrm{~Hz}, 3 \mathrm{H}) .{ }^{\mathrm{B}} \mathrm{C}\left\{{ }^{\prime} \mathrm{H}\right\} \mathrm{NMR}(126$ $\left.\mathrm{MHz}, \mathrm{CDCl}_{3}, 25^{\circ} \mathrm{C}, \delta\right): 60.0,49.7,43.6,40.9,31.9,29.5,29.3,28.7,28.3,22.8,14.2$. Anal. Calcd for $\mathrm{C}_{13} \mathrm{H}_{28} \mathrm{OS}_{2}$ : C, 59.04; H, 10.67. Found: C, 58.87; H, 11.01 .<smiles>COC(=O)CCSSCC(C)C</smiles>

Spectroscopic data of methyl 3-(tert-butyldisulfaneyl)propanoate (2oc): ${ }^{\text {ss }}$ Column chromatography $\left(\mathrm{SiO}_{2}\right.$, hexane/dichloromethane=4/1 to $\left.1 / 1, \mathrm{v} / \mathrm{v}\right)$ afforded the desired product (127 mg, 60\%) as a colorless oil. ${ }^{\mathrm{H}} \mathrm{NMR}\left(500 \mathrm{MHz}, \mathrm{CDCl}_{3}, 25{ }^{\circ} \mathrm{C}, \delta\right): 3.70(\mathrm{~s}$, $3 \mathrm{H}), 2.93(\mathrm{t}, J=7.3 \mathrm{~Hz}, 2 \mathrm{H}), 2.72(\mathrm{t}, J=7.3 \mathrm{~Hz}, 2 \mathrm{H}), 1.34(\mathrm{~s}, 9 \mathrm{H}) .{ }^{\mathrm{l}} \mathrm{C}\left\{{ }^{\prime} \mathrm{H}\right\} \mathrm{NMR}(126$ $\left.\mathrm{MHz}, \mathrm{CDCl}_{3}, 25^{\circ} \mathrm{C}, \delta\right): 172.3,51.9,48.0,34.9,34.1,30.0$.<smiles>CC(C)(CCO)SSC1CCCCC1</smiles>

Spectroscopic data of 3-(cyclohexyldisulfany)-3-methylbutan-1-ol (2ek): Column chromatography $\left(\mathrm{SiO}_{2}\right.$, hexane/ethyl acetate $=100 / 0$ to $\left.7 / 3, \mathrm{v} / \mathrm{v}\right)$ afforded the desired product $\left(158 \mathrm{mg}, 67 \%\right.$ ) as a colorless oil. IR (neat, $\left.\mathrm{cm}^{-1}\right) 3347,2929,2852,1447 .{ }^{\mathrm{H}}$ $\operatorname{NMR}\left(500 \mathrm{MHz}, \mathrm{CDCl}_{3}, 25{ }^{\circ} \mathrm{C}, \delta\right): 3.79(\mathrm{t}, J=7.0 \mathrm{~Hz}, 2 \mathrm{H}), 2.73-2.68(\mathrm{~m}, 1 \mathrm{H})$, 2.11-2.03 (m, 2H), $1.89(\mathrm{t}, J=7.0 \mathrm{~Hz}, 2 \mathrm{H}), 1.82-1.73(\mathrm{~m}, 2 \mathrm{H}), 1.64-1.58(\mathrm{~m}, 1 \mathrm{H}), 1.52$ (br s, $1 \mathrm{H}), 1.35-1.18(\mathrm{~m}, 11 \mathrm{H}) .{ }^{\mathrm{i}} \mathrm{C}\left\{{ }^{\prime} \mathrm{H}\right\} \mathrm{NMR}\left(126 \mathrm{MHz}, \mathrm{CDCl}_{3}, 25^{\circ} \mathrm{C}, \delta\right): 60.0,50.3$, 49.3, 43.7, 33.0, 28.3 , 26.1, 25.8. Anal. Calcd for $\mathrm{C}_{11} \mathrm{H}_{22} \mathrm{OS}$ : C, 56.36; H, 9.46. Found: $\mathrm{C}$, $56.04 ; \mathrm{H}, 9.57$.

Measurements of Nanosecond Laser Flash Spectroscopy. The transient absorption spectra of $\mathbf{F l}$ in the presence of $\mathbf{1 l}$ were measured under nitrogen atmosphere using the following system. A solution of $\mathbf{F l}(0.12 \mathrm{mM})$ and $\mathbf{1 l}(13 \mathrm{mM})$ in $\mathrm{MeOH} / \mathrm{H}_{2} \mathrm{O} /$ pyridine $(9: 1: 0.05, \mathrm{v} / \mathrm{v}, 3 \mathrm{~mL})$ was set in a sealed quartz cell $\left(10 \times 10 \times 40 \mathrm{~mm}^{3}\right)$ after removing molecular oxygen by freeze-pump-thaw cycle. The sample solution was irradiated by a laser pulse at $355 \mathrm{~nm}$ with $6 \mathrm{~ns}$ duration of a Nd:YAG laser (GCR150, Spectra Physics). 
The repetition rate of the laser pulses was $10 \mathrm{~Hz}$. A Xe flash lamp with $2 \mu$ s duration (L4633, Hamamatsu Photonics) was used as the probe light source. The probe light transmitted through the laser-irradiated part of the sample solution was conducted into a monochrometer (Kymera-193i, Andor Technology) through glass fiber and was captured by an intensified charge-coupled device detector (ICCD; DH334T, Andor Technology). The width of the ICCD gate was set at 100 ns. A digital pulse-delay generator (DG535, Stanford Research Systems) was used to synchronize the laser, the flash lamp, and the ICCD gate and adjust the delay time between the laser pulse and the ICCD gate. The difference absorption spectra shown in Figure 2 were obtained by subtracting an absorption spectrum collected without laser excitation from absorption spectra collected after laser excitation.

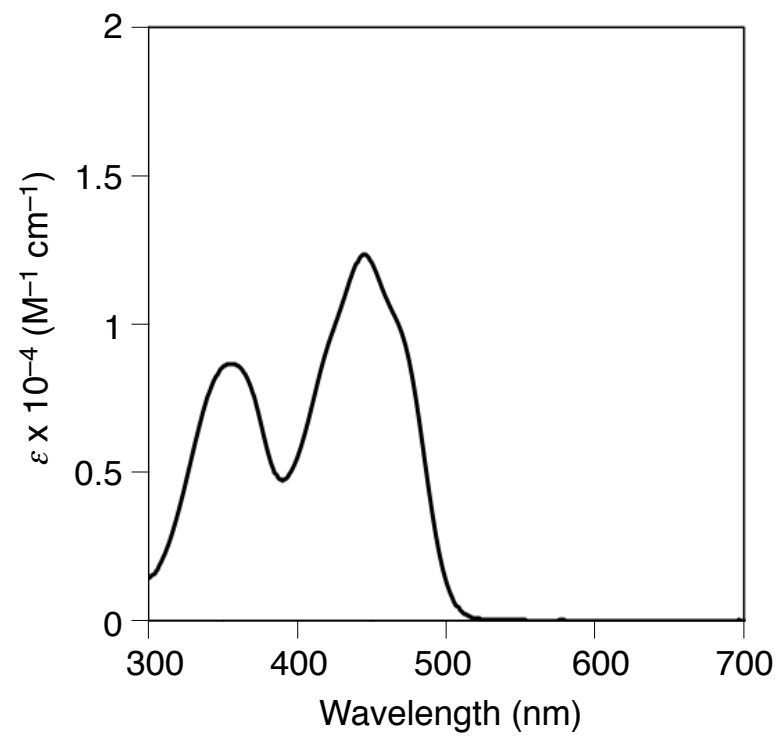

Figure S1. Absorption spectra of $\mathbf{F l}$ in $\mathrm{MeOH} / \mathrm{H}_{2} \mathrm{O} /$ pyridine (9:1:0.05, v/v, $0.12 \mathrm{mM}$ ). 
Optimization of Coupling Reaction. The catalytic activity of diverse flavin compounds was investigated for the homocoupling of $\mathbf{1 b}$ as shown in Table $\mathrm{S} 1$. In this reaction condition, the best efficiency was achieved by $\mathbf{F l}$.

Table S1. Effect of flavin catalysts on homocoupling of $\mathbf{1 b}$.
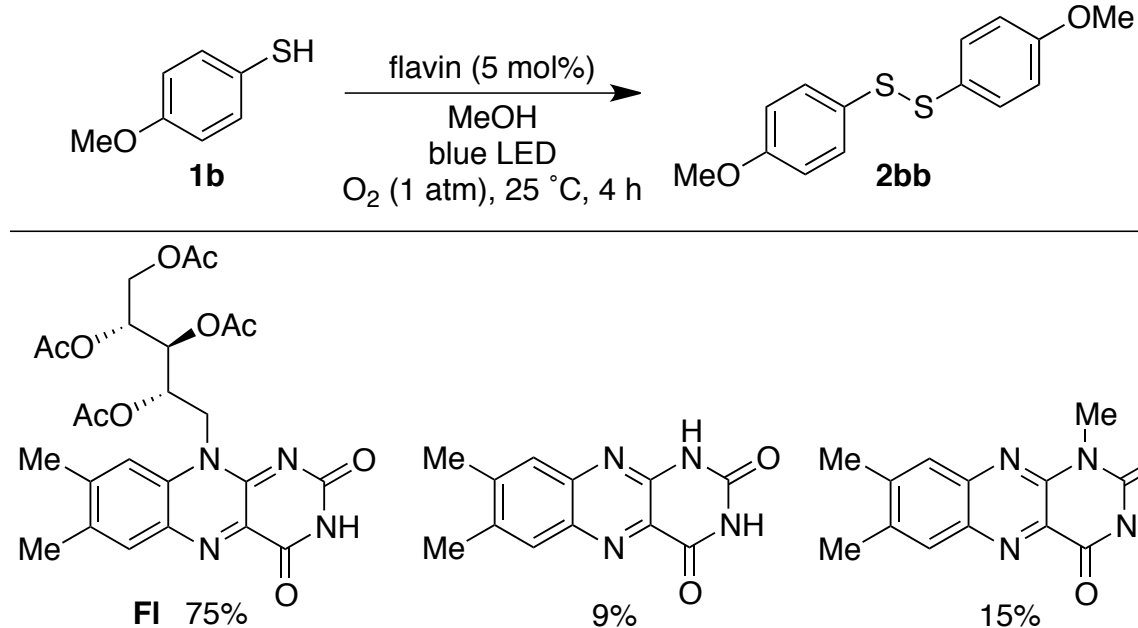<smiles>Cc1cc2nc3[nH]c(=O)[nH]c(=O)c3nc2cc1C</smiles><smiles>[Y6]n1c(=O)n(C)c(=O)c2nc3cc(C)c(C)cc3nc21</smiles><smiles></smiles><smiles></smiles><smiles></smiles>

Conditions: $1 \mathrm{~b}(0.01 \mathrm{M})$, flavin $(5 \mathrm{~mol} \%)$, and $\mathrm{MeOH}$ under $\mathrm{O}_{2}(1 \mathrm{~atm})$ at $25{ }^{\circ} \mathrm{C}$ for $4 \mathrm{~h}$. The yield was determined by GC. 
4. 'H and ${ }^{\mathrm{B}} \mathrm{C}\left\{{ }^{\prime} \mathrm{H}\right\}$ NMR Spectra of Novel Compounds<smiles>CC(C)(CCO)SSc1ccccn1</smiles>

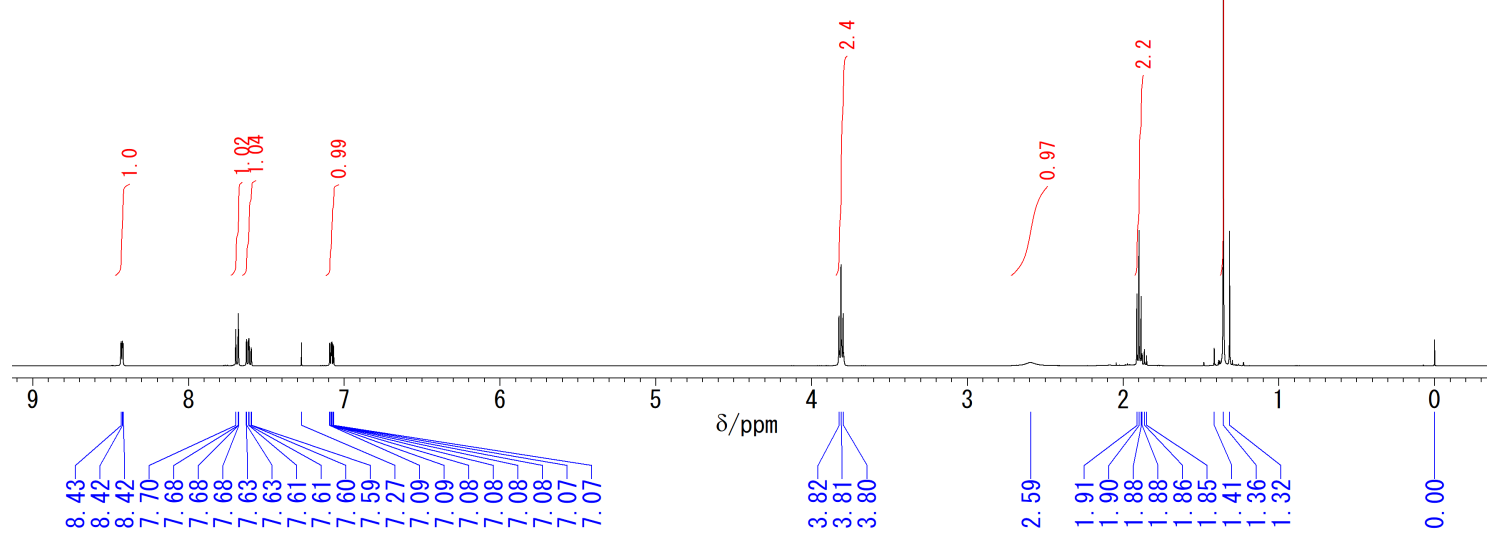

Spectrum S1. ${ }^{\mathrm{H}} \mathrm{NMR}\left(\mathrm{CDCl}_{3}, 500 \mathrm{MHz}\right)$ spectrum of compound 2 ae.

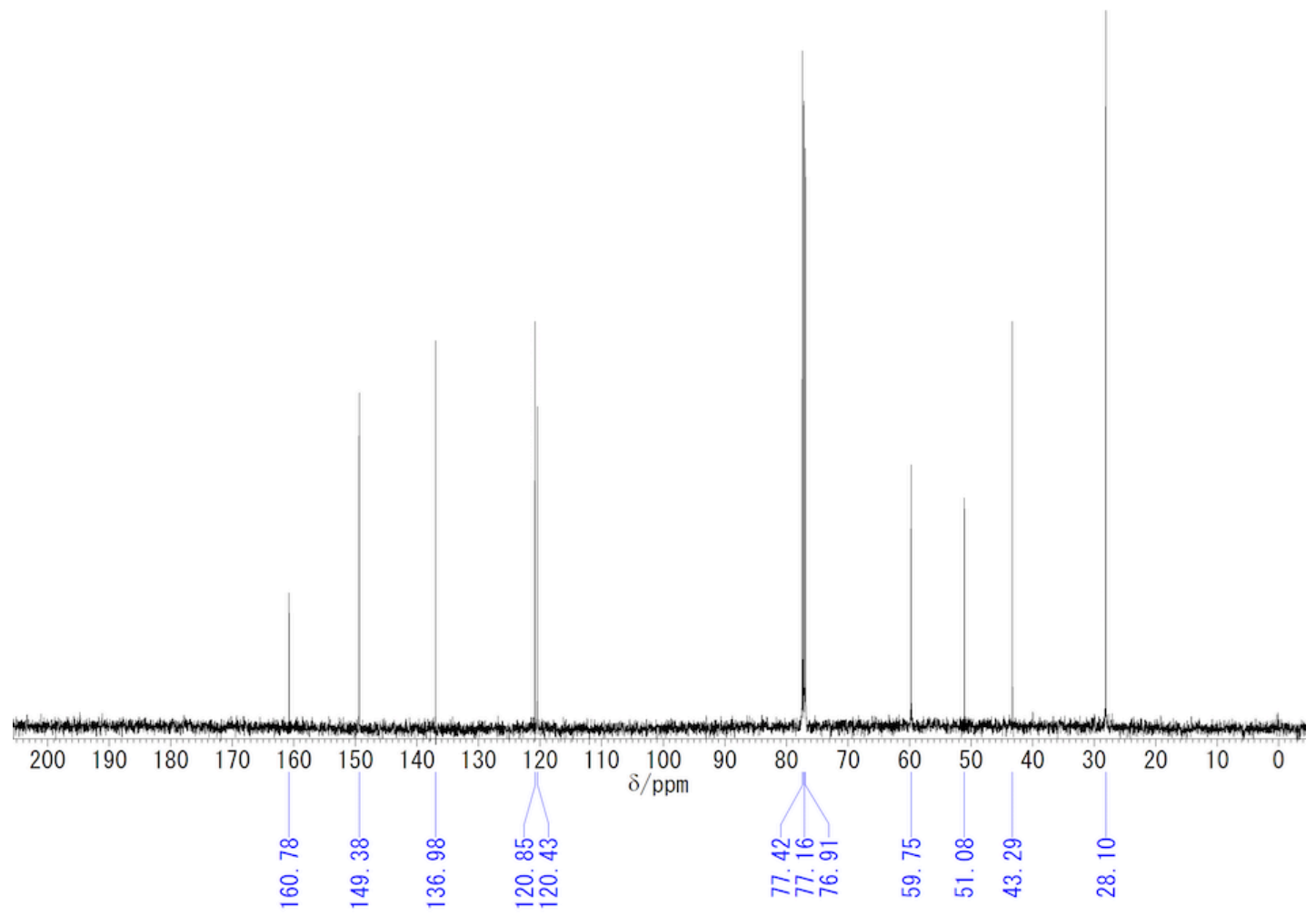

Spectrum S2. ${ }^{1} \mathrm{C}$ NMR $\left(\mathrm{CDCl}_{3}, 126 \mathrm{MHz}\right)$ spectrum of compound 2ae. 
<smiles>CC(C)(CCO)SSc1ccccc1</smiles>

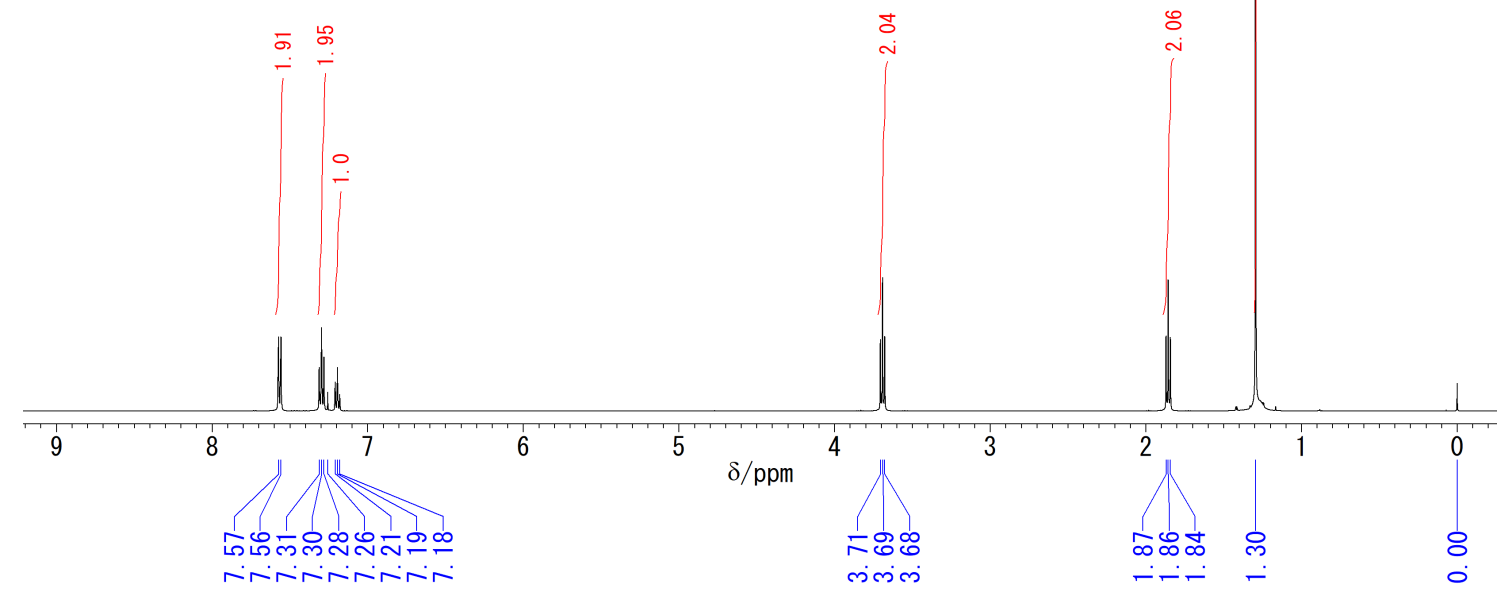

Spectrum S3. ${ }^{\mathrm{H}} \mathrm{NMR}\left(\mathrm{CDCl}_{3}, 500 \mathrm{MHz}\right)$ spectrum of compound 2ie.

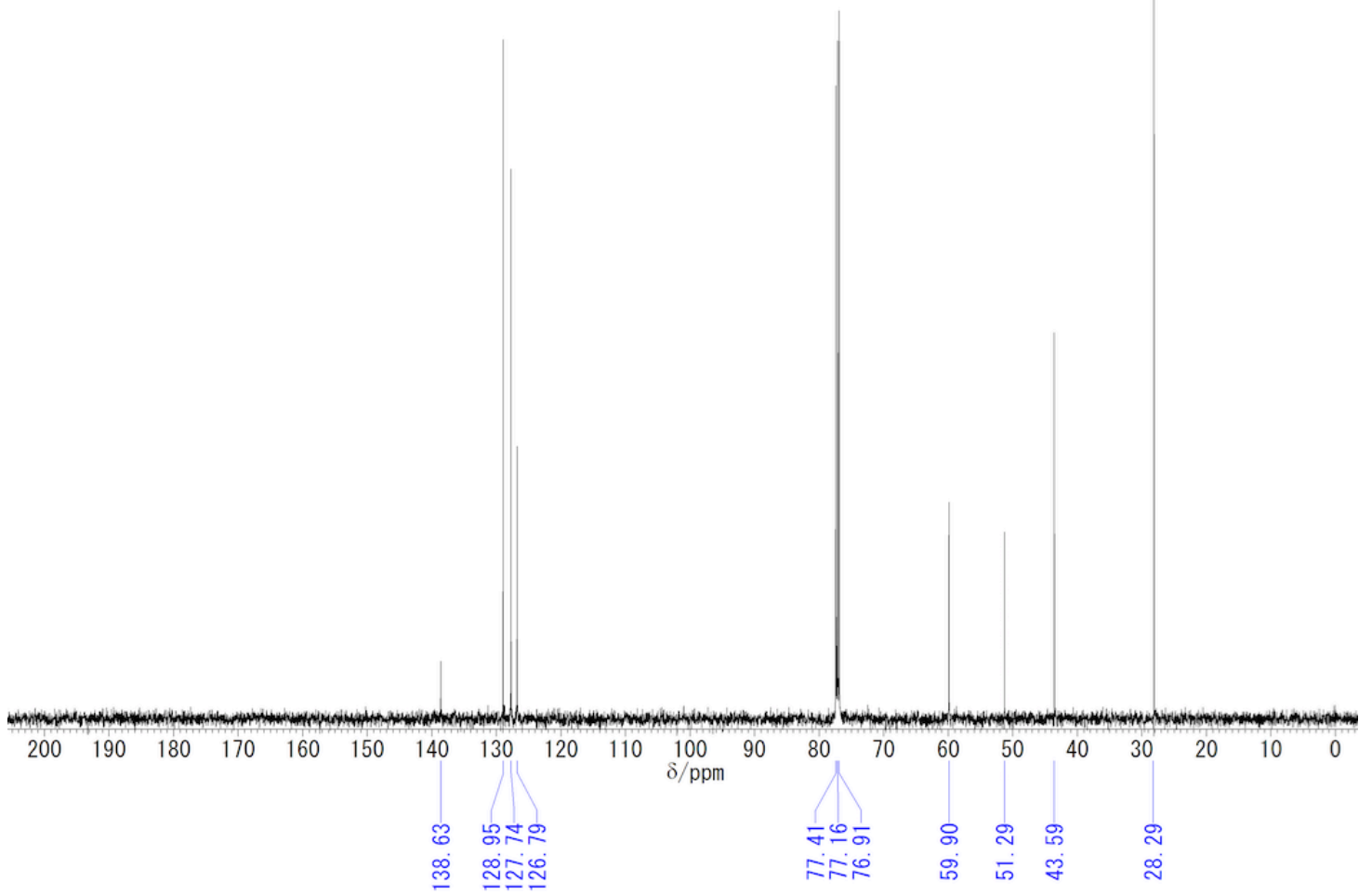

Spectrum S4. ${ }^{\mathrm{B}} \mathrm{C} \mathrm{NMR}\left(\mathrm{CDCl}_{3}, 126 \mathrm{MHz}\right)$ spectrum of compound 2 ie. 


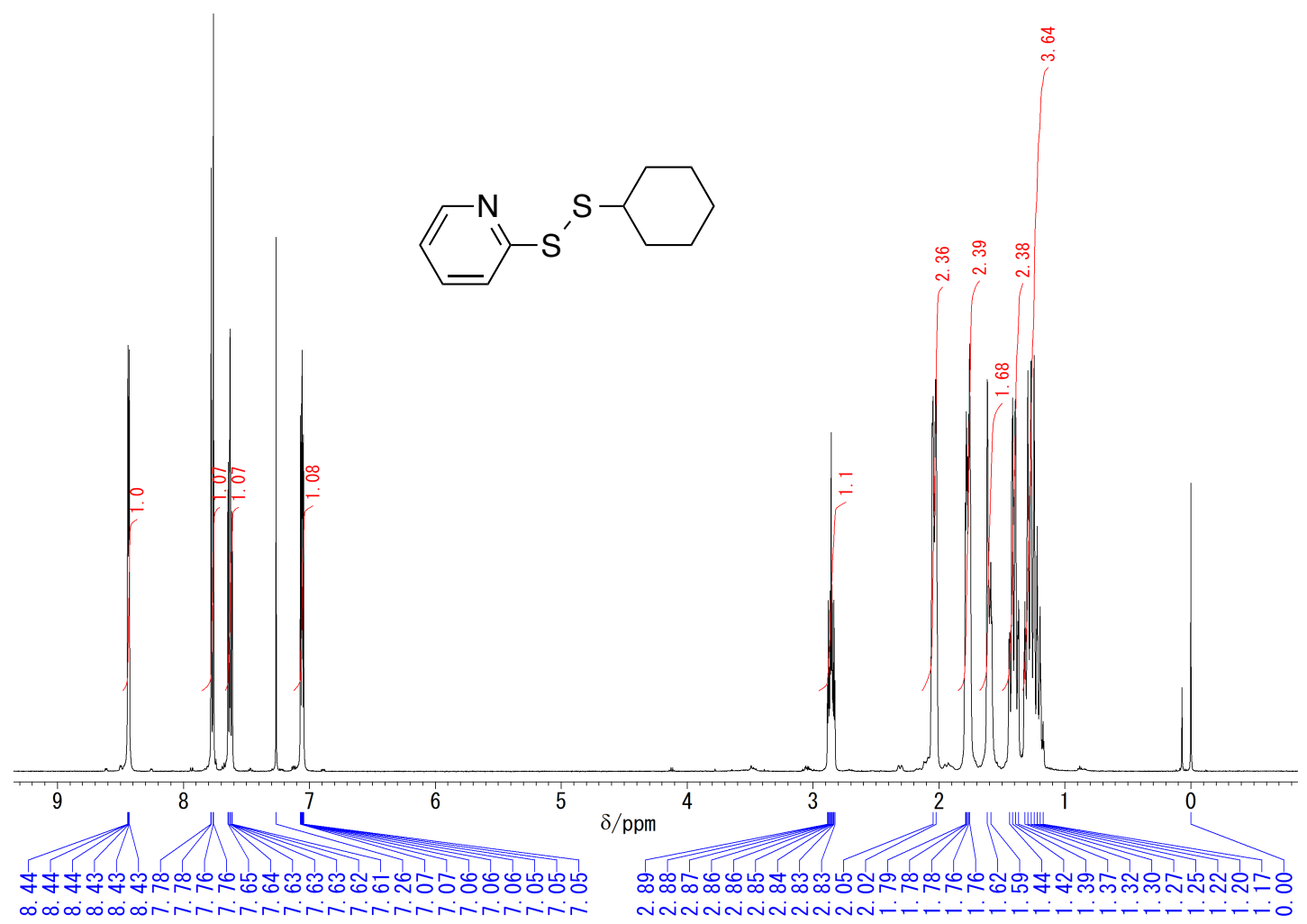

Spectrum S5. 'H NMR $\left(\mathrm{CDCl}_{3}, 500 \mathrm{MHz}\right)$ spectrum of compound 2ak.

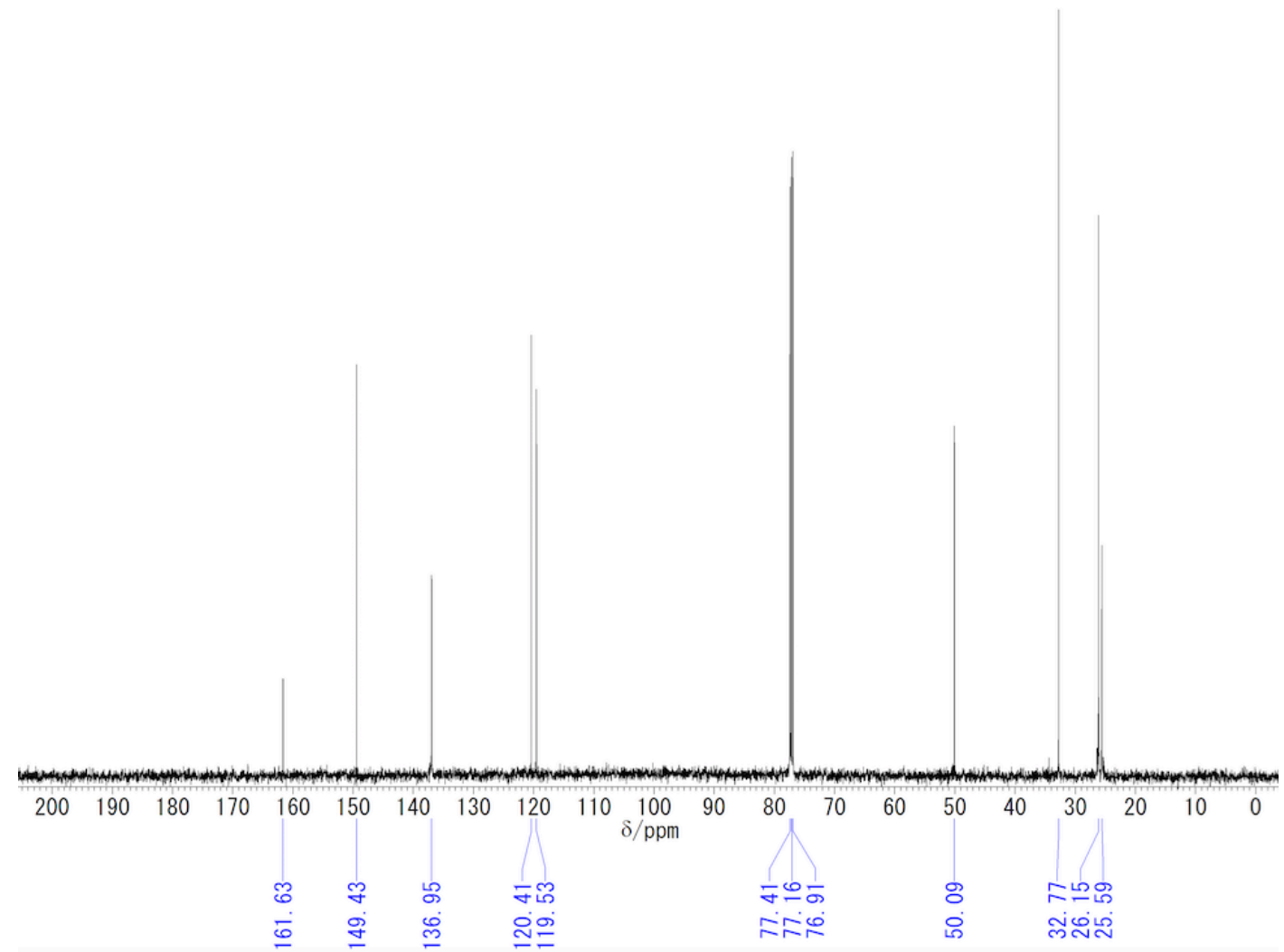

Spectrum S6. ${ }^{13} \mathrm{C}$ NMR $\left(\mathrm{CDCl}_{3}, 126 \mathrm{MHz}\right)$ spectrum of compound 2ak. 


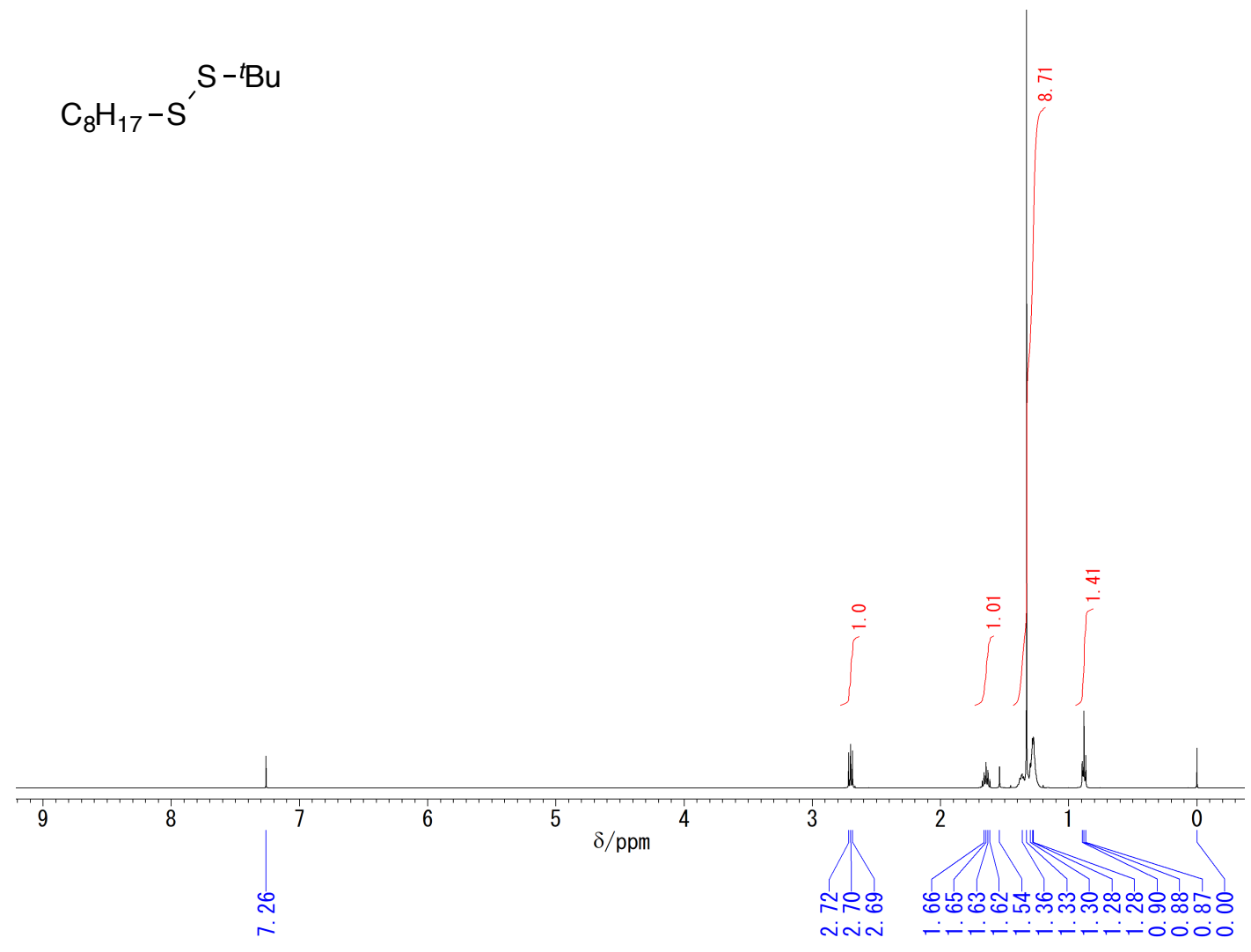

Spectrum S7. ${ }^{\mathrm{H}} \mathrm{NMR}\left(\mathrm{CDCl}_{3}, 500 \mathrm{MHz}\right)$ spectrum of compound 2lc.

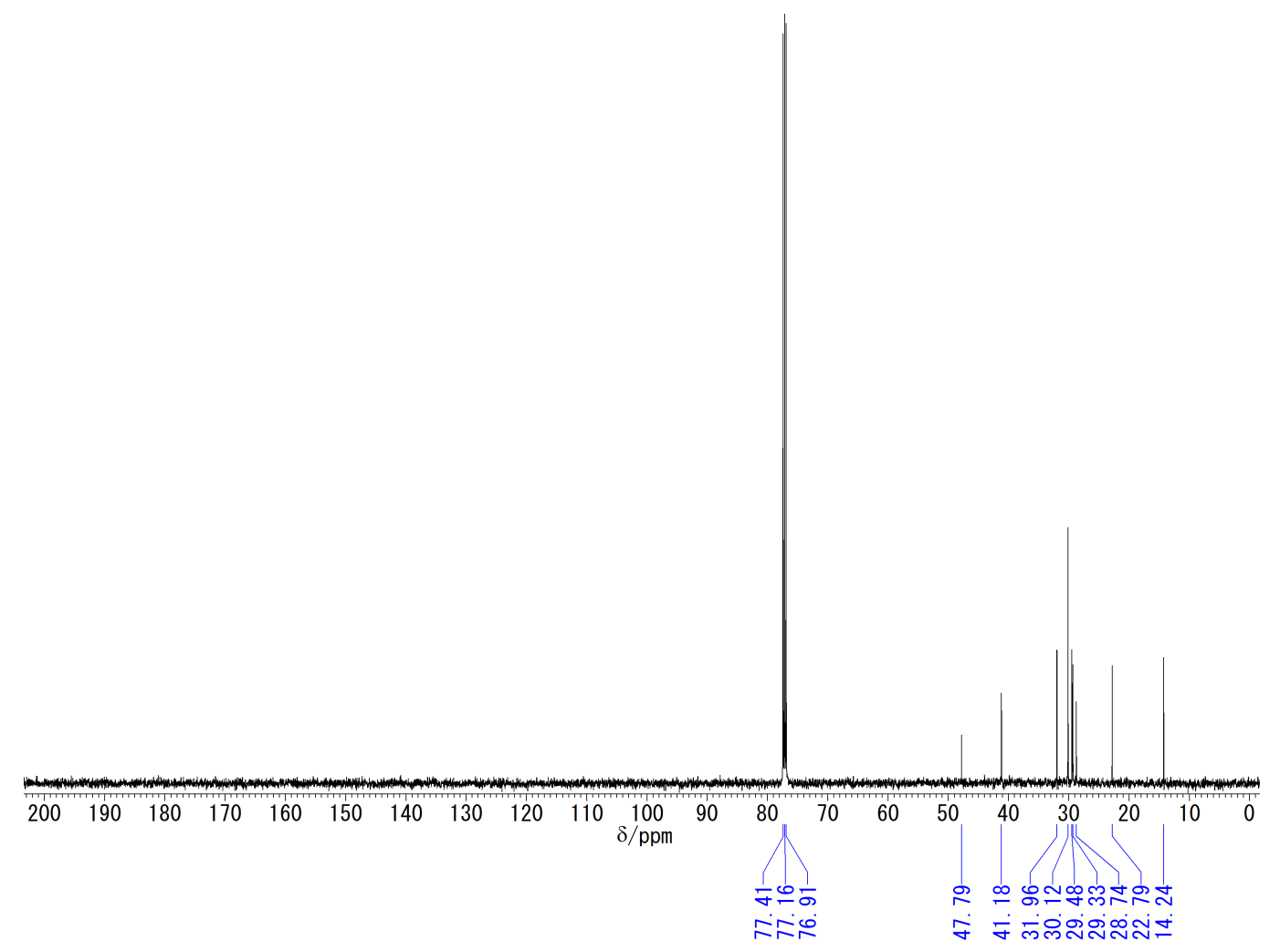

Spectrum S8. ${ }^{13} \mathrm{C}$ NMR $\left(\mathrm{CDCl}_{3}, 126 \mathrm{MHz}\right)$ spectrum of compound 2lc. 


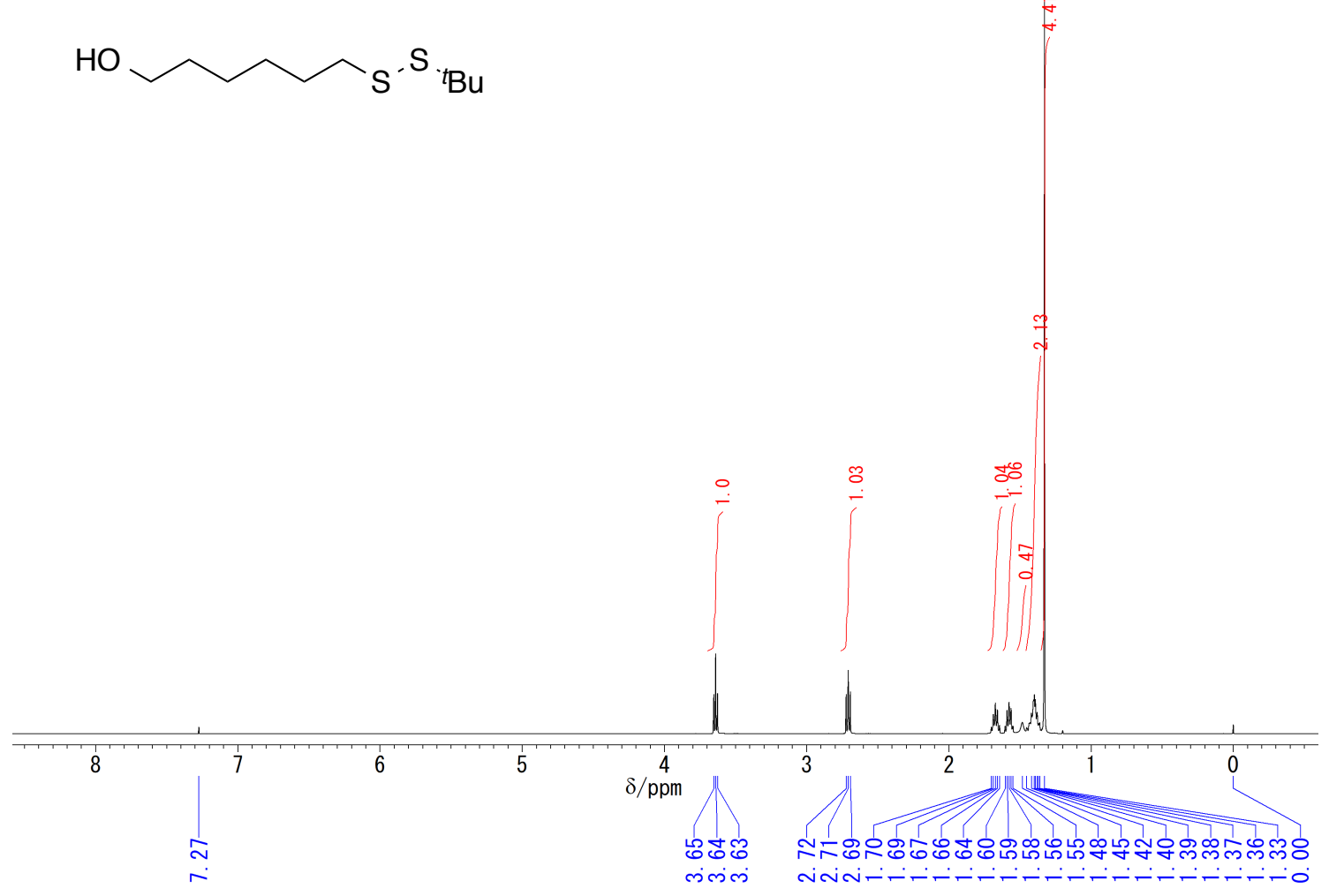

Spectrum S9. 'H NMR $\left(\mathrm{CDCl}_{3}, 500 \mathrm{MHz}\right)$ spectrum of compound 2 mc.

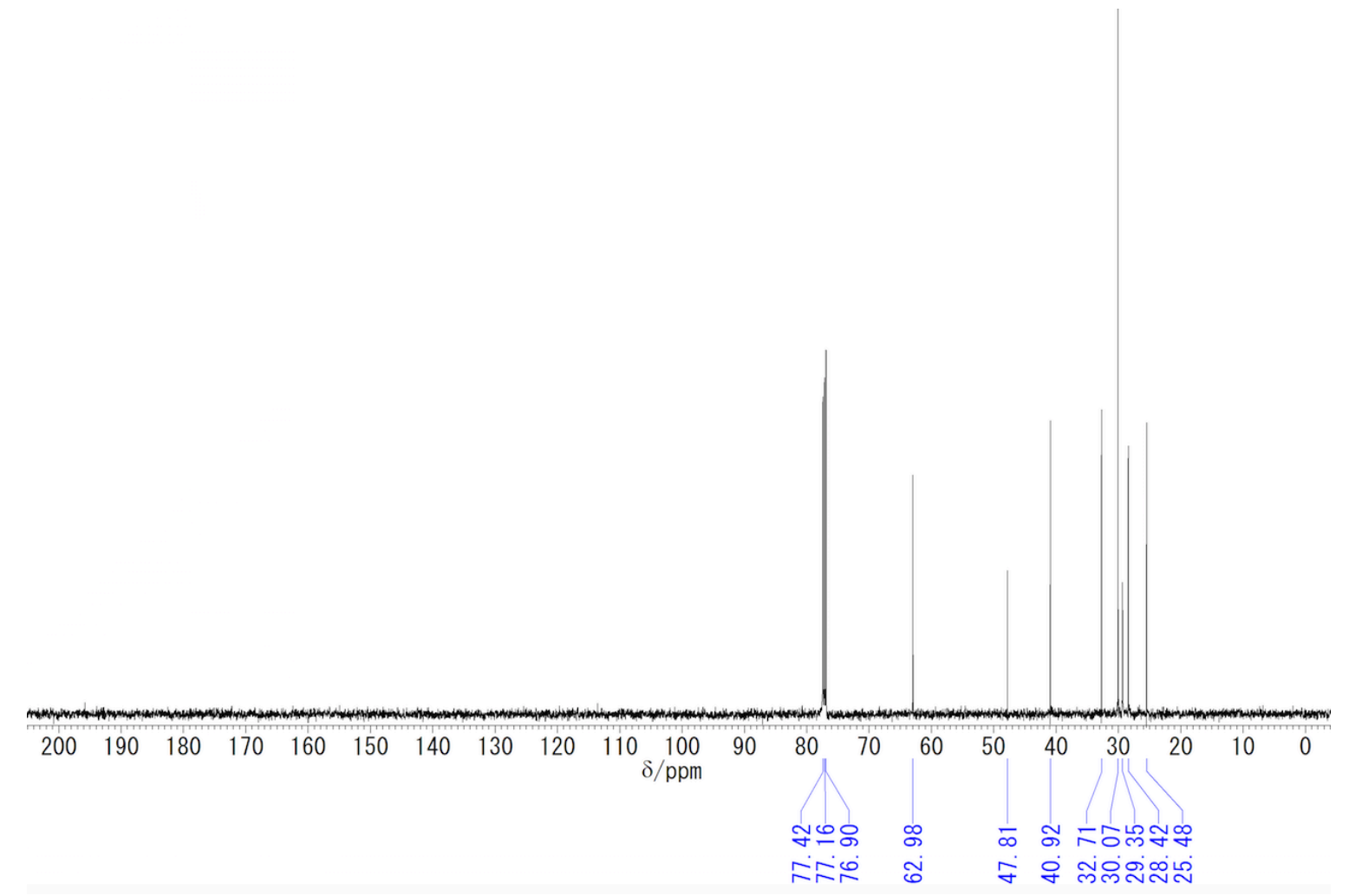

Spectrum S10. ${ }^{\circledR \mathrm{C}} \mathrm{NMR}\left(\mathrm{CDCl}_{3}, 126 \mathrm{MHz}\right)$ spectrum of compound $\mathbf{2 m c}$. 


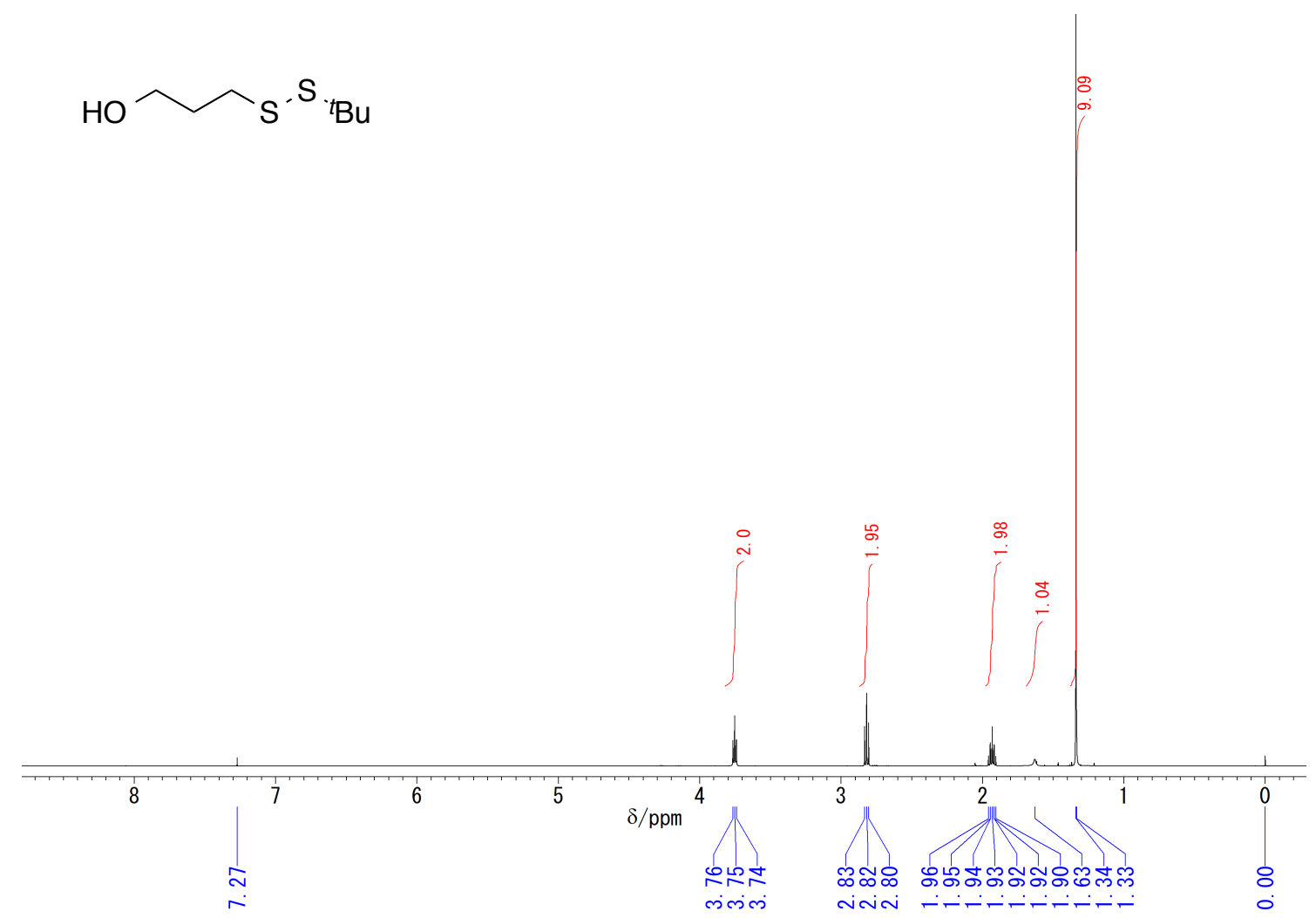

Spectrum S11. 'H NMR $\left(\mathrm{CDCl}_{3}, 500 \mathrm{MHz}\right)$ spectrum of compound 2nc.

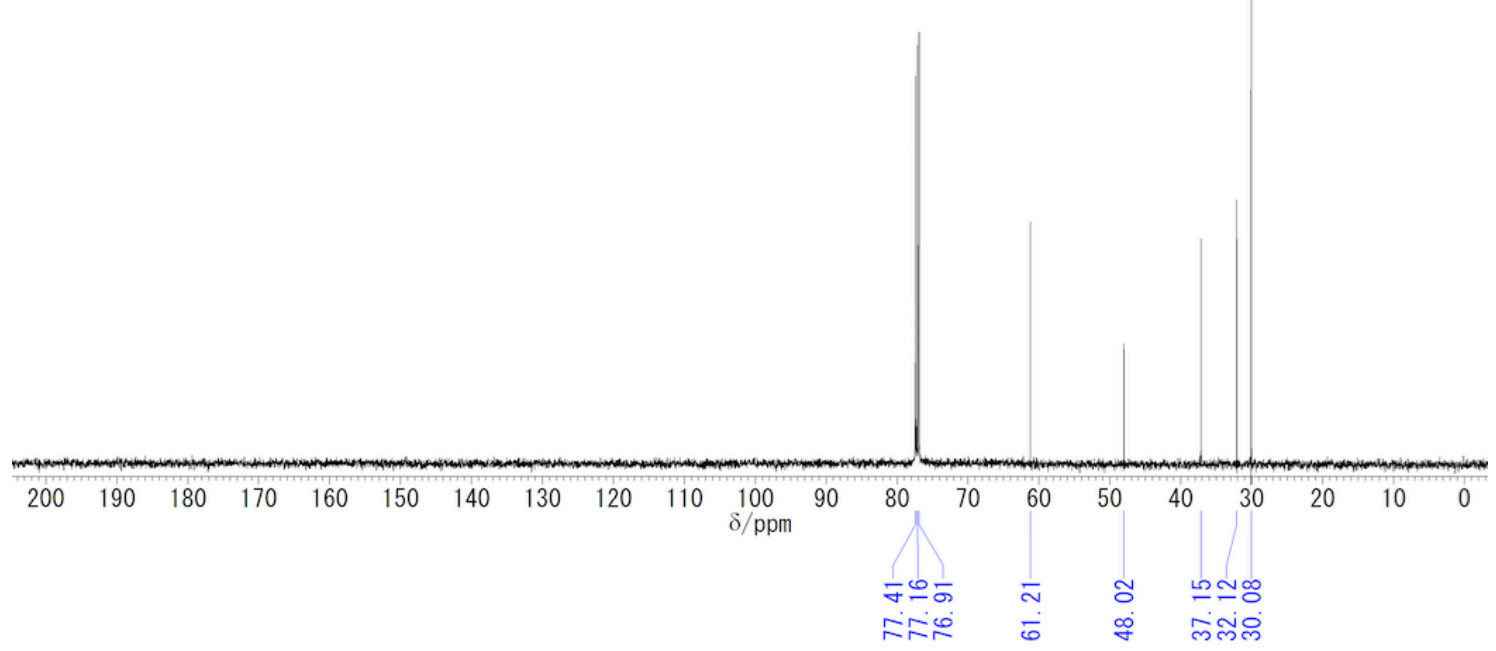

Spectrum S12. ${ }^{13} \mathrm{C}$ NMR $\left(\mathrm{CDCl}_{3}, 126 \mathrm{MHz}\right)$ spectrum of compound 2nc. 


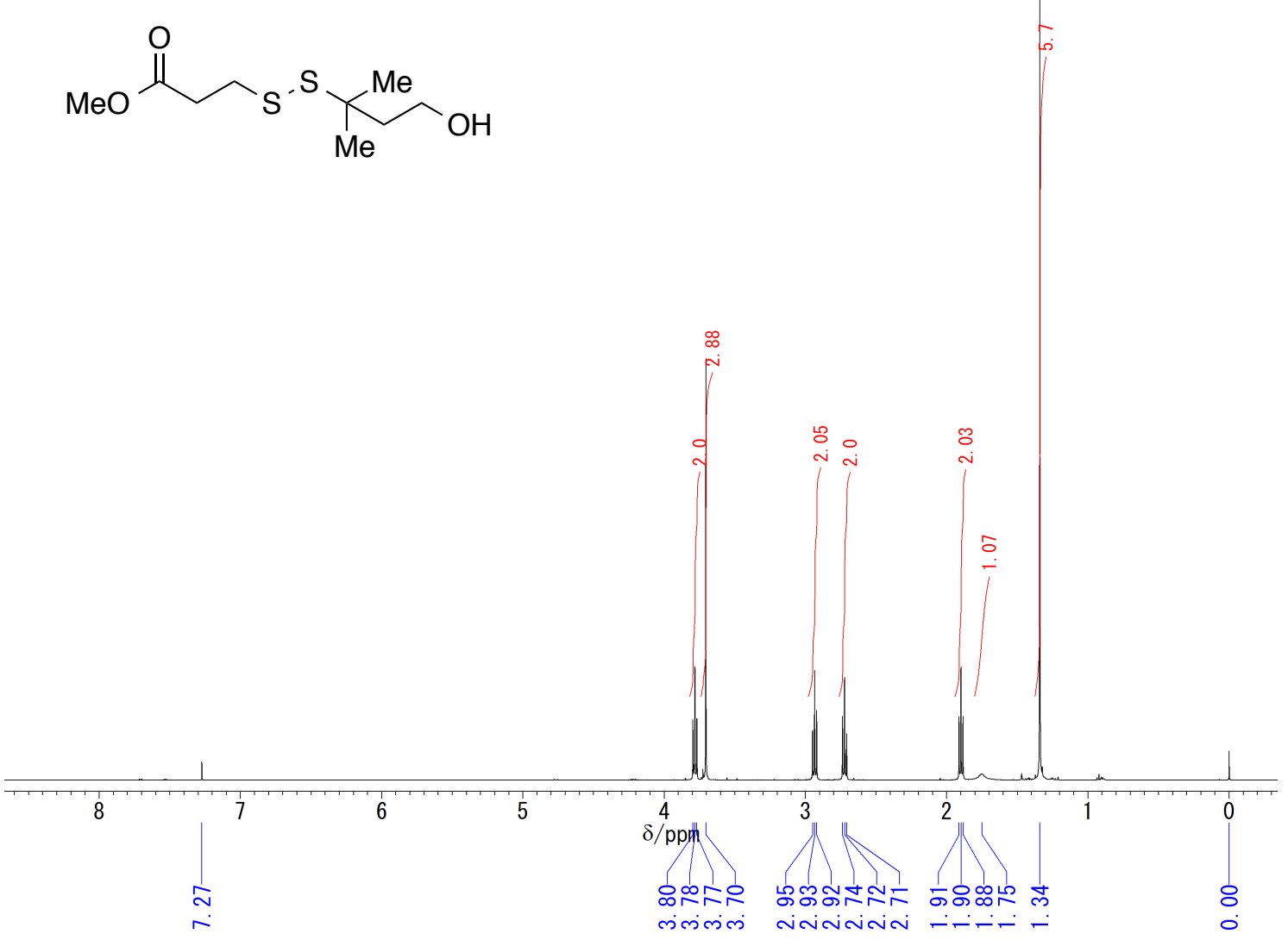

Spectrum S13. 'H NMR $\left(\mathrm{CDCl}_{3}, 500 \mathrm{MHz}\right)$ spectrum of compound 20 .

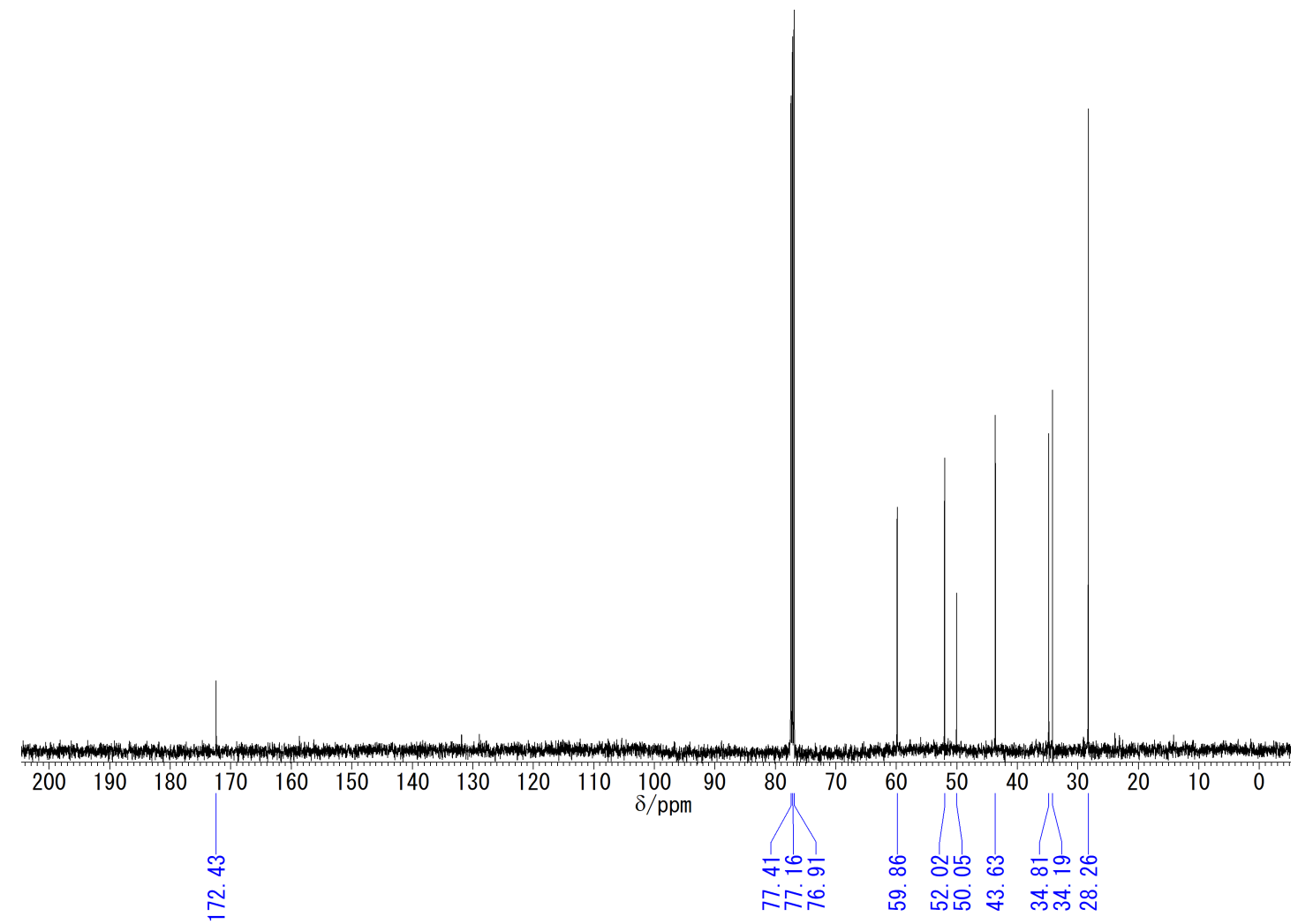

Spectrum S14. ${ }^{13} \mathrm{C}$ NMR $\left(\mathrm{CDCl}_{3}, 126 \mathrm{MHz}\right)$ spectrum of compound $20 e$. 


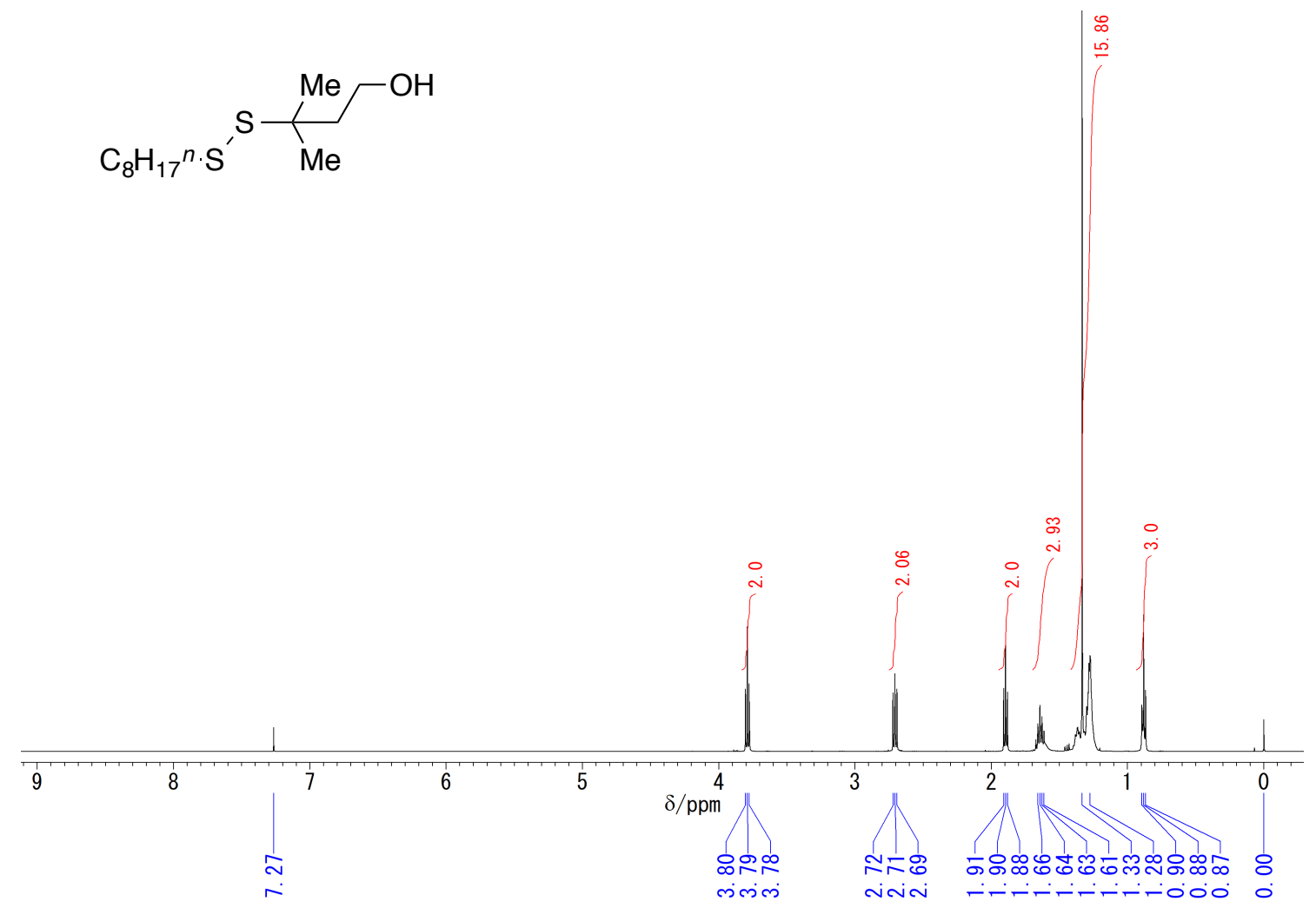

Spectrum S15. 'H NMR ( $\left.\mathrm{CDCl}_{3}, 500 \mathrm{MHz}\right)$ spectrum of compound 2 le.

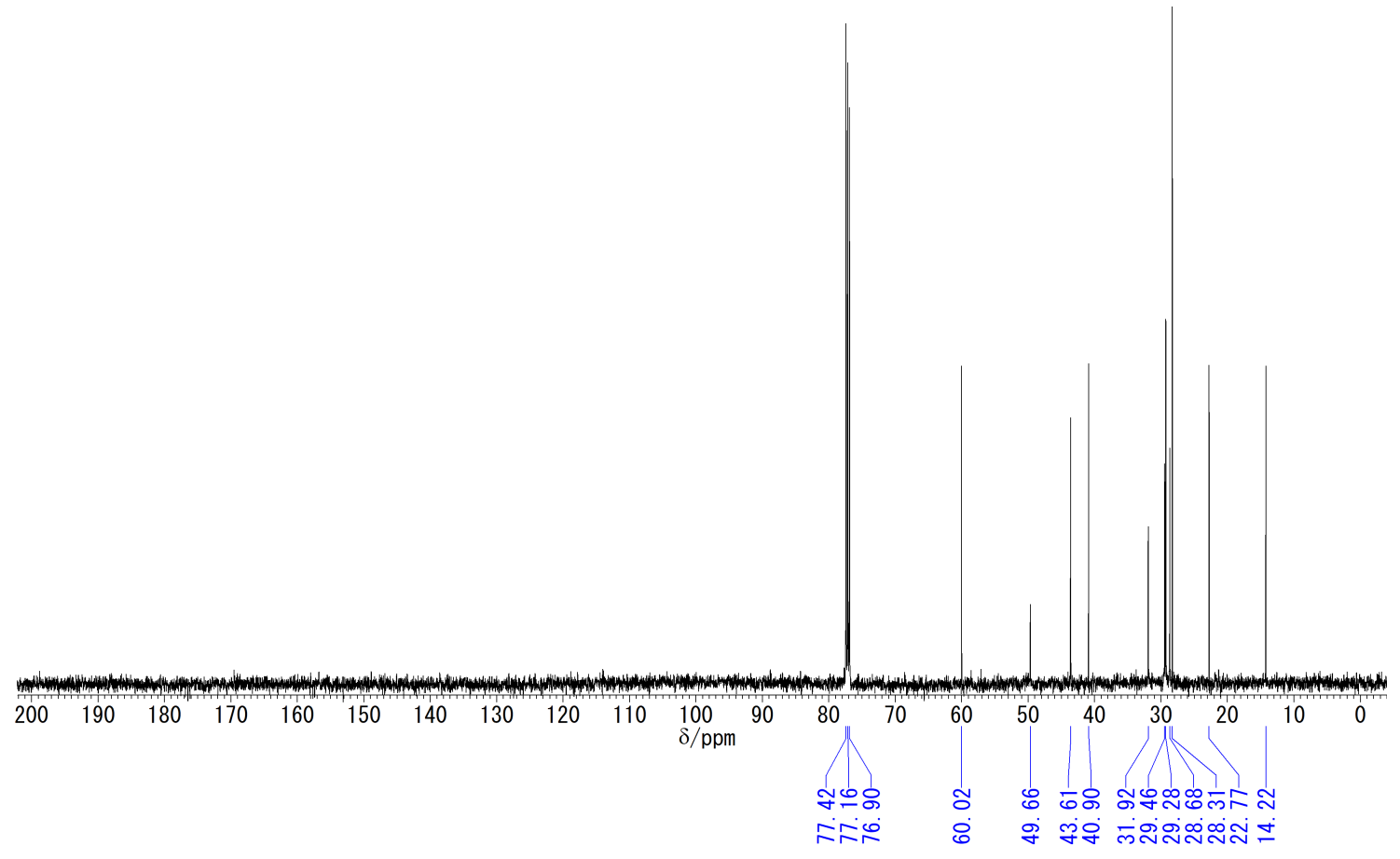

Spectrum S16. ${ }^{13} \mathrm{C} \mathrm{NMR}\left(\mathrm{CDCl}_{3}, 126 \mathrm{MHz}\right)$ spectrum of compound 2 le. 


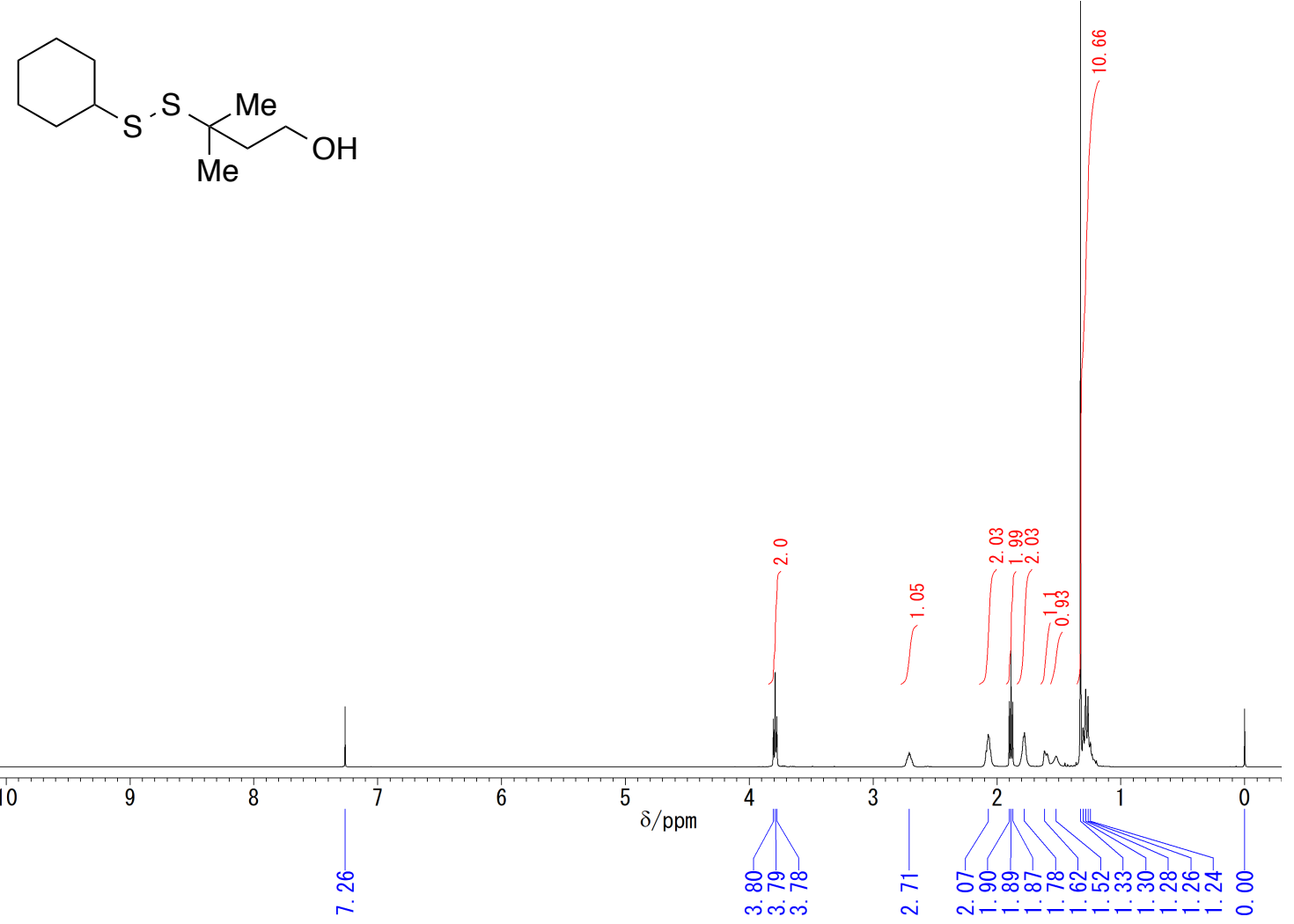

Spectrum S17. 'H NMR $\left(\mathrm{CDCl}_{3}, 500 \mathrm{MHz}\right)$ spectrum of compound 2ek.

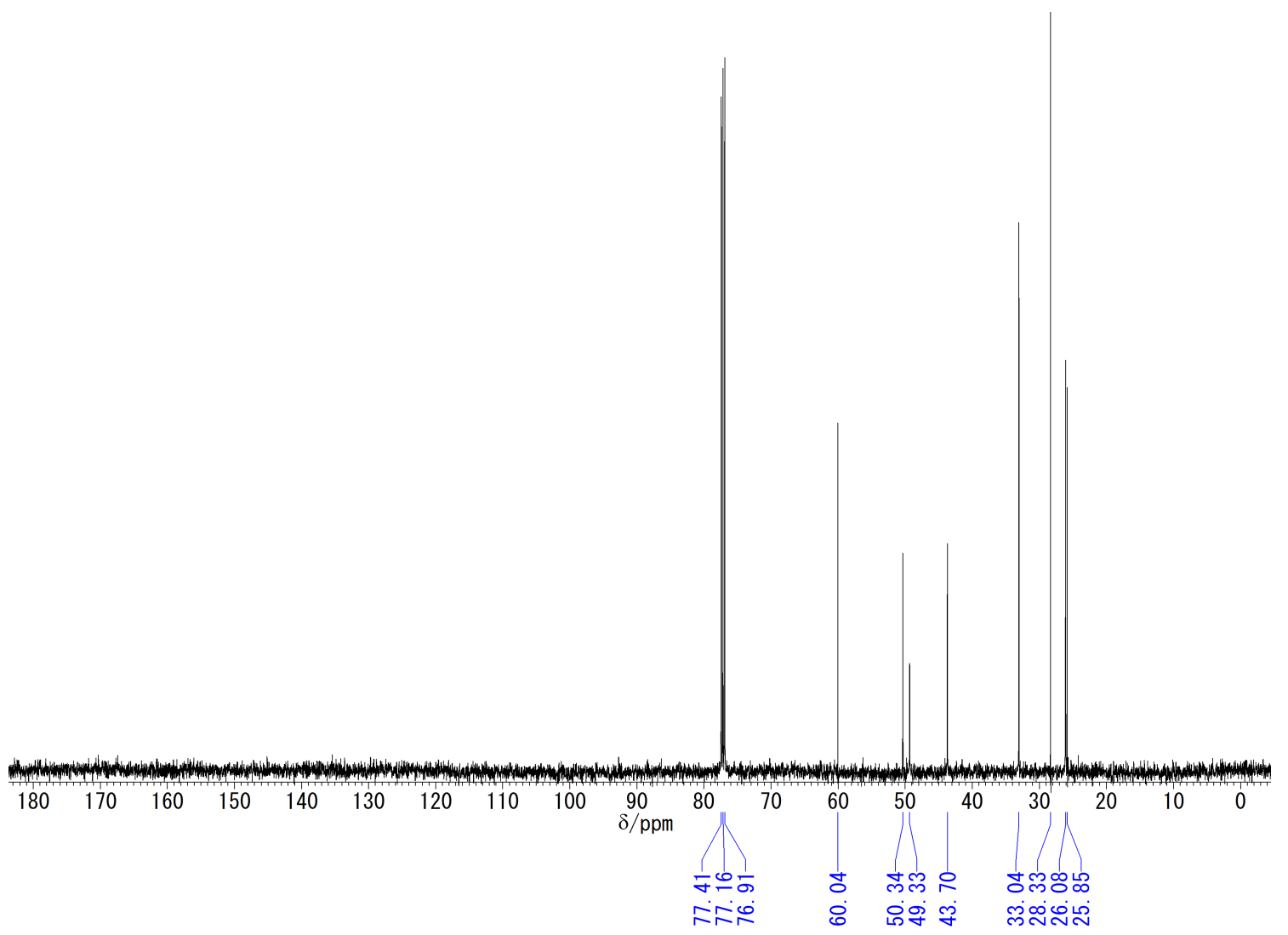

Spectrum S18. ${ }^{13} \mathrm{C}$ NMR $\left(\mathrm{CDCl}_{3}, 126 \mathrm{MHz}\right)$ spectrum of compound 2ek. 


\section{5. 'H NMR Spectra of Known Compounds}<smiles>CC(C)CSSc1ccccn1</smiles>
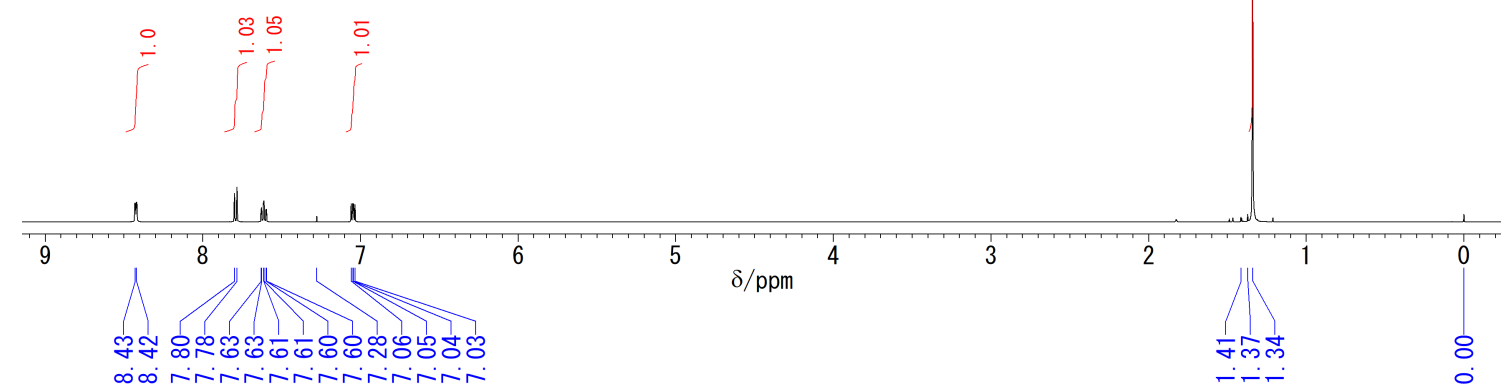

Spectrum S19. 'H NMR $\left(\mathrm{CDCl}_{3}, 500 \mathrm{MHz}\right)$ spectrum of compound 2 ac.<smiles>CC(C)(C)SSSc1ccncc1</smiles>

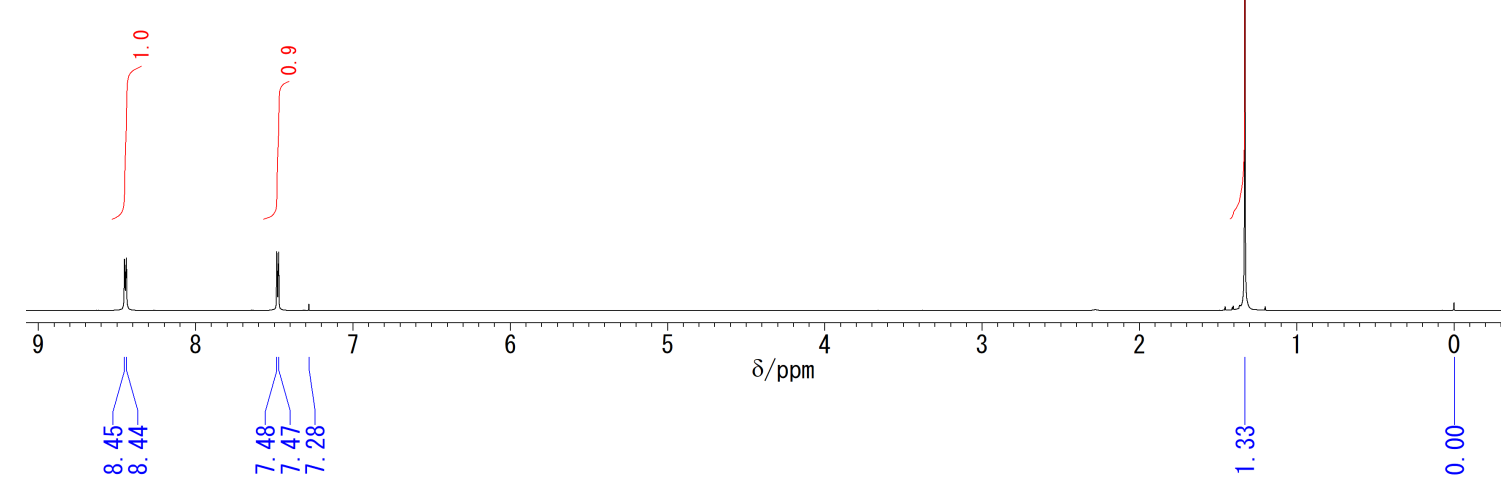

Spectrum S20. 'H NMR $\left(\mathrm{CDCl}_{3}, 500 \mathrm{MHz}\right)$ spectrum of compound 2 dc. 
<smiles>CCSSc1ncccn1</smiles>

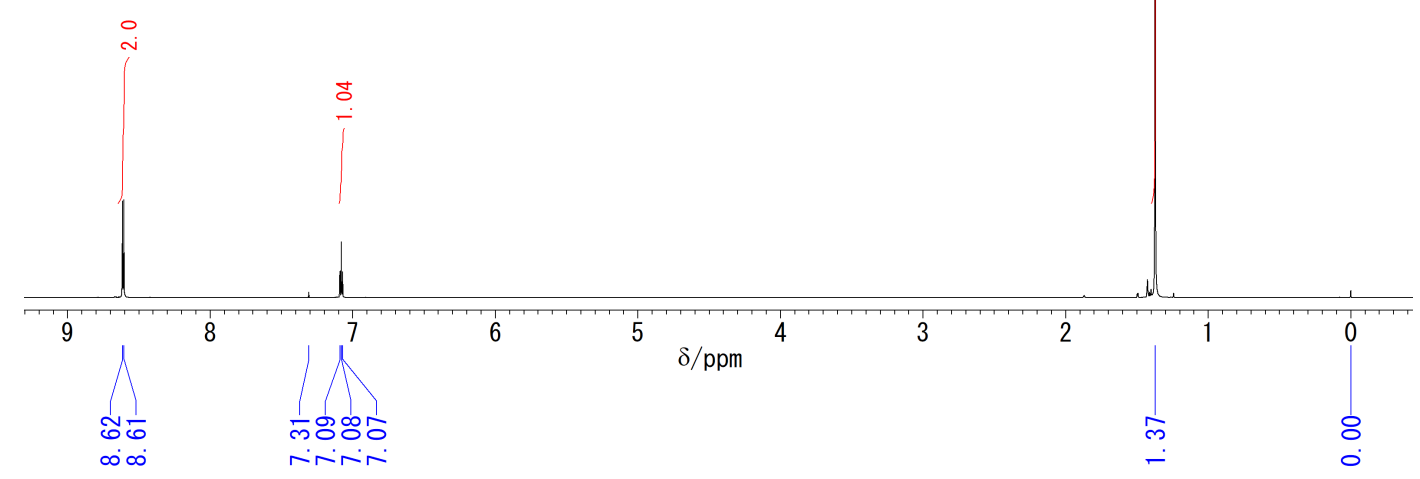

Spectrum S21. 'H NMR ( $\left.\mathrm{CDCl}_{3}, 500 \mathrm{MHz}\right)$ spectrum of compound $2 \mathbf{f c}$.<smiles>CC(C)SSc1nc2ccccc2s1</smiles>

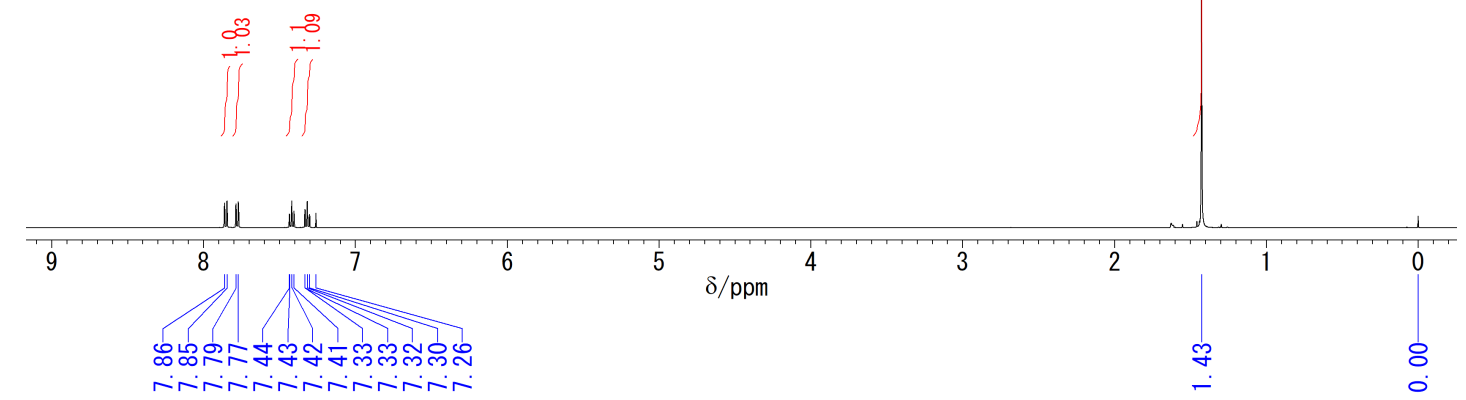

Spectrum S22. 'H NMR $\left(\mathrm{CDCl}_{3}, 500 \mathrm{MHz}\right)$ spectrum of compound $2 \mathrm{gc}$. 


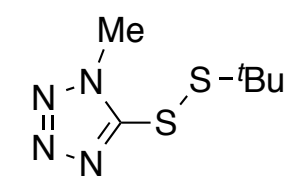

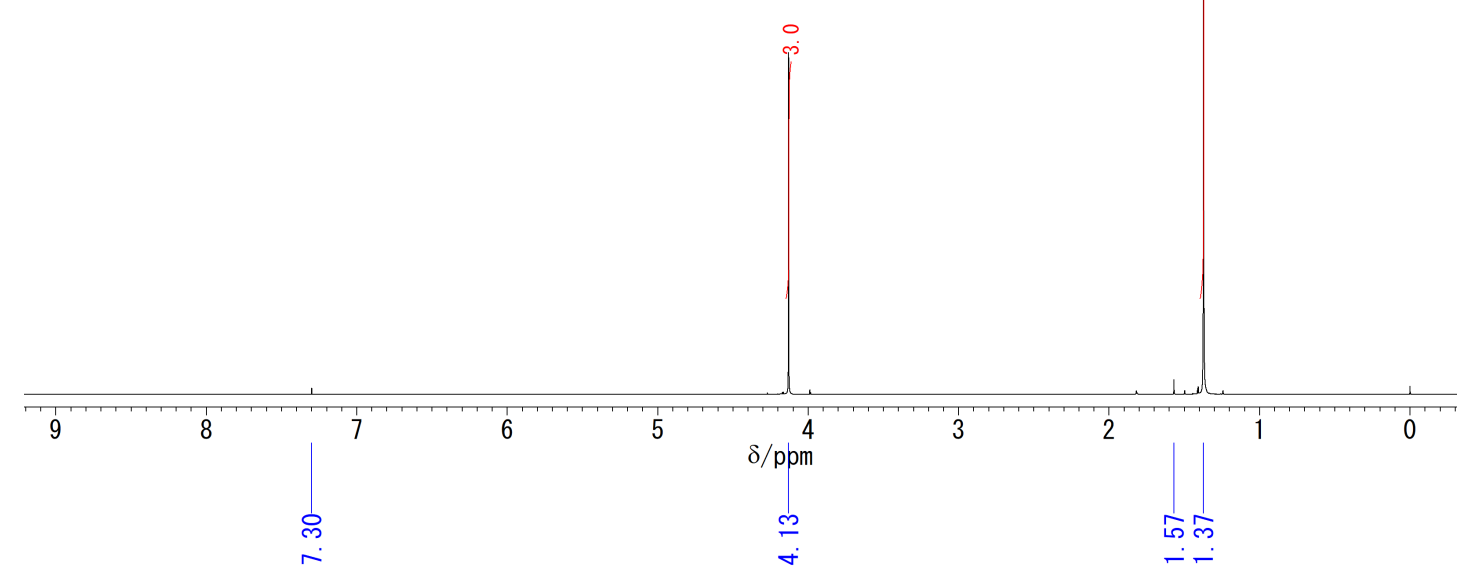

Spectrum S23. 'H NMR ( $\left.\mathrm{CDCl}_{3}, 500 \mathrm{MHz}\right)$ spectrum of compound 2 hc.<smiles>CC(C)CSSc1ccc(Cl)cc1</smiles>

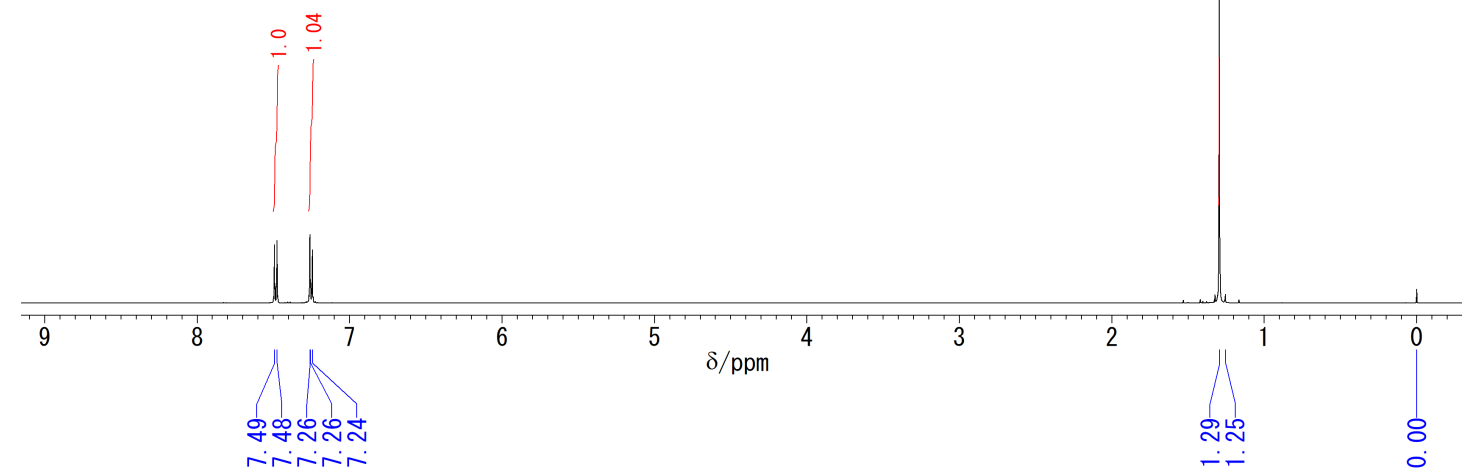

Spectrum S24. ${ }^{1} \mathrm{H}$ NMR $\left(\mathrm{CDCl}_{3}, 500 \mathrm{MHz}\right)$ spectrum of compound $\mathbf{2 j c}$. 


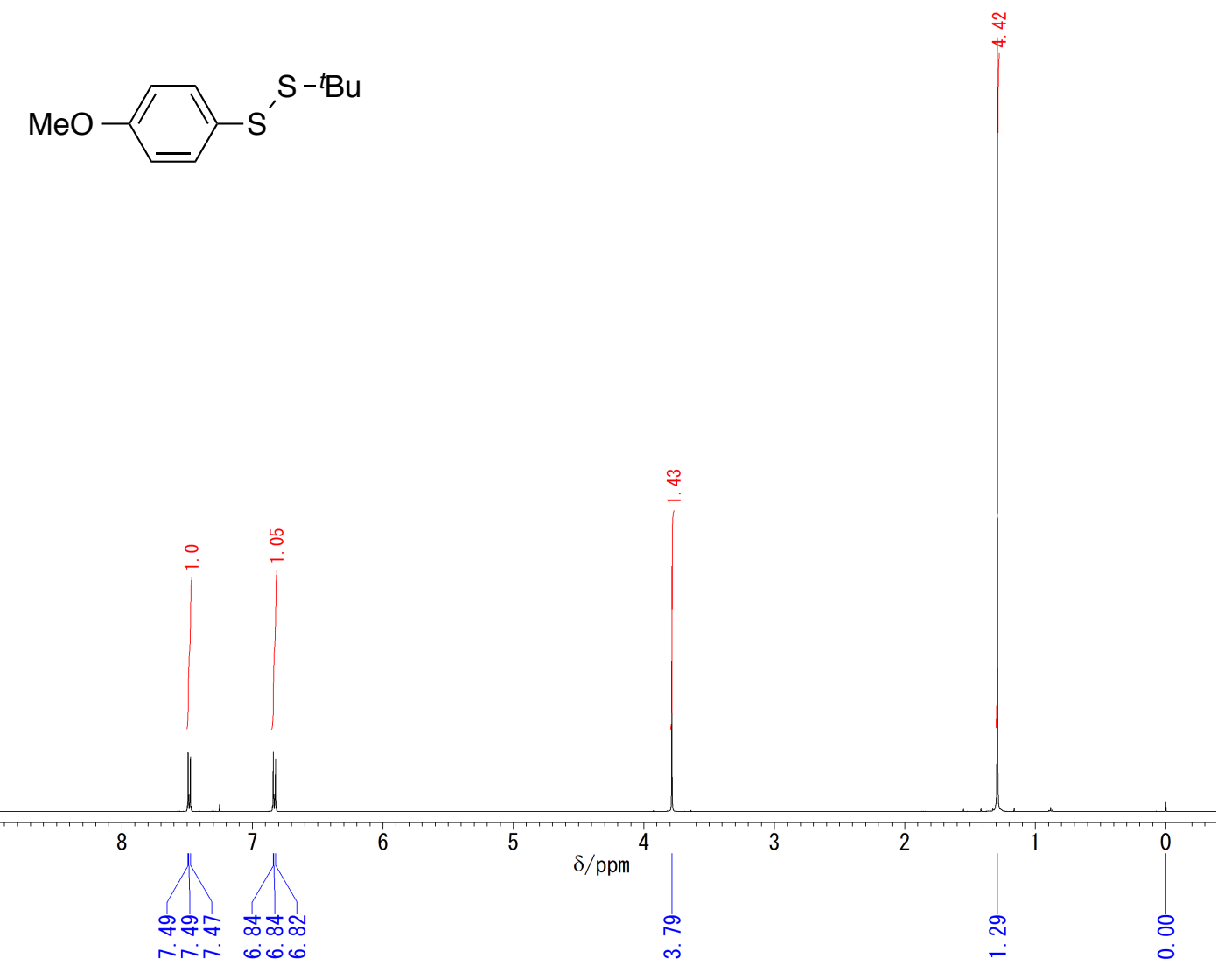

Spectrum S25. 'H NMR ( $\left.\mathrm{CDCl}_{3}, 500 \mathrm{MHz}\right)$ spectrum of compound 2 bc.

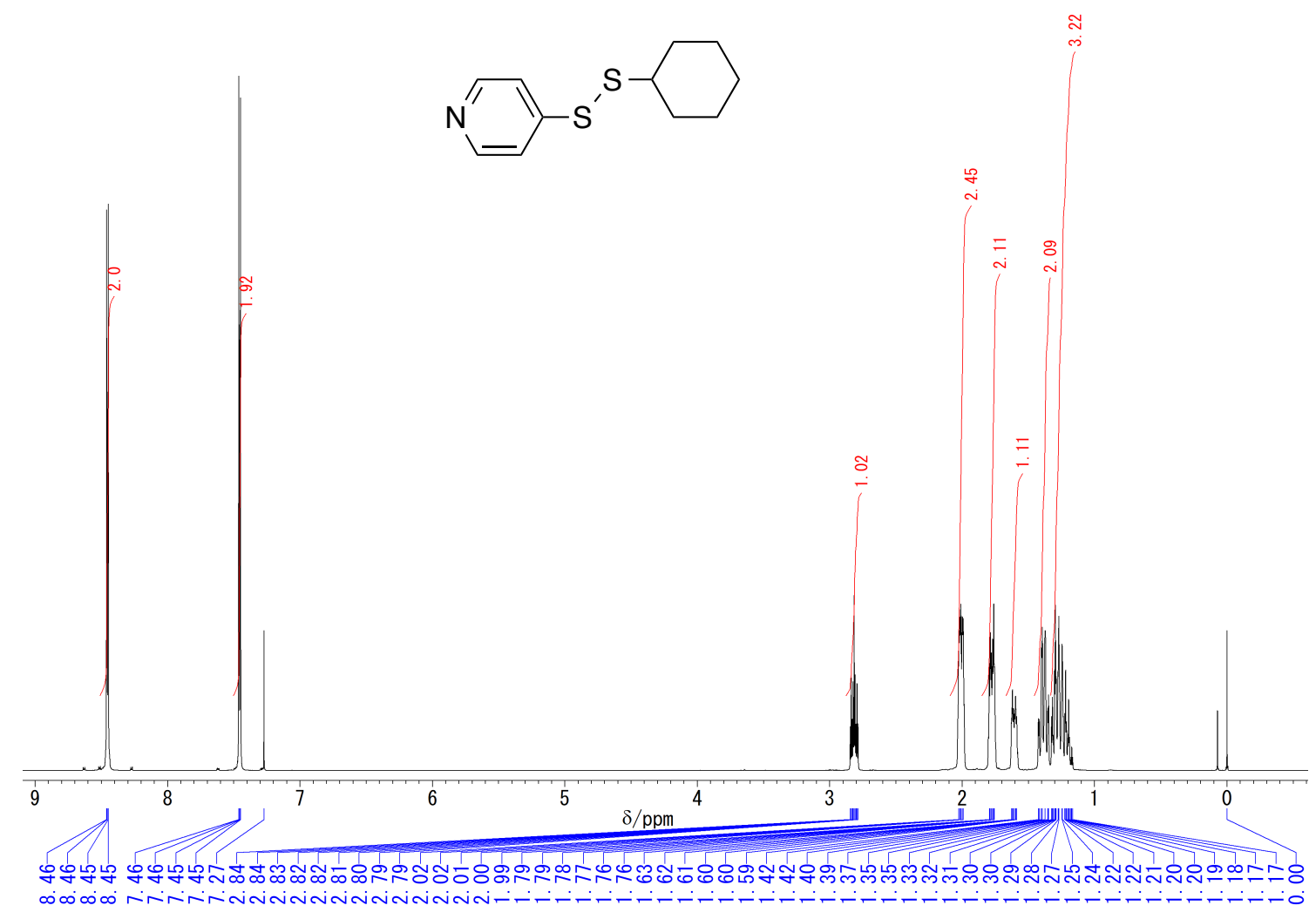

Spectrum S26. 'H NMR $\left(\mathrm{CDCl}_{3}, 500 \mathrm{MHz}\right)$ spectrum of compound 2 dk. 


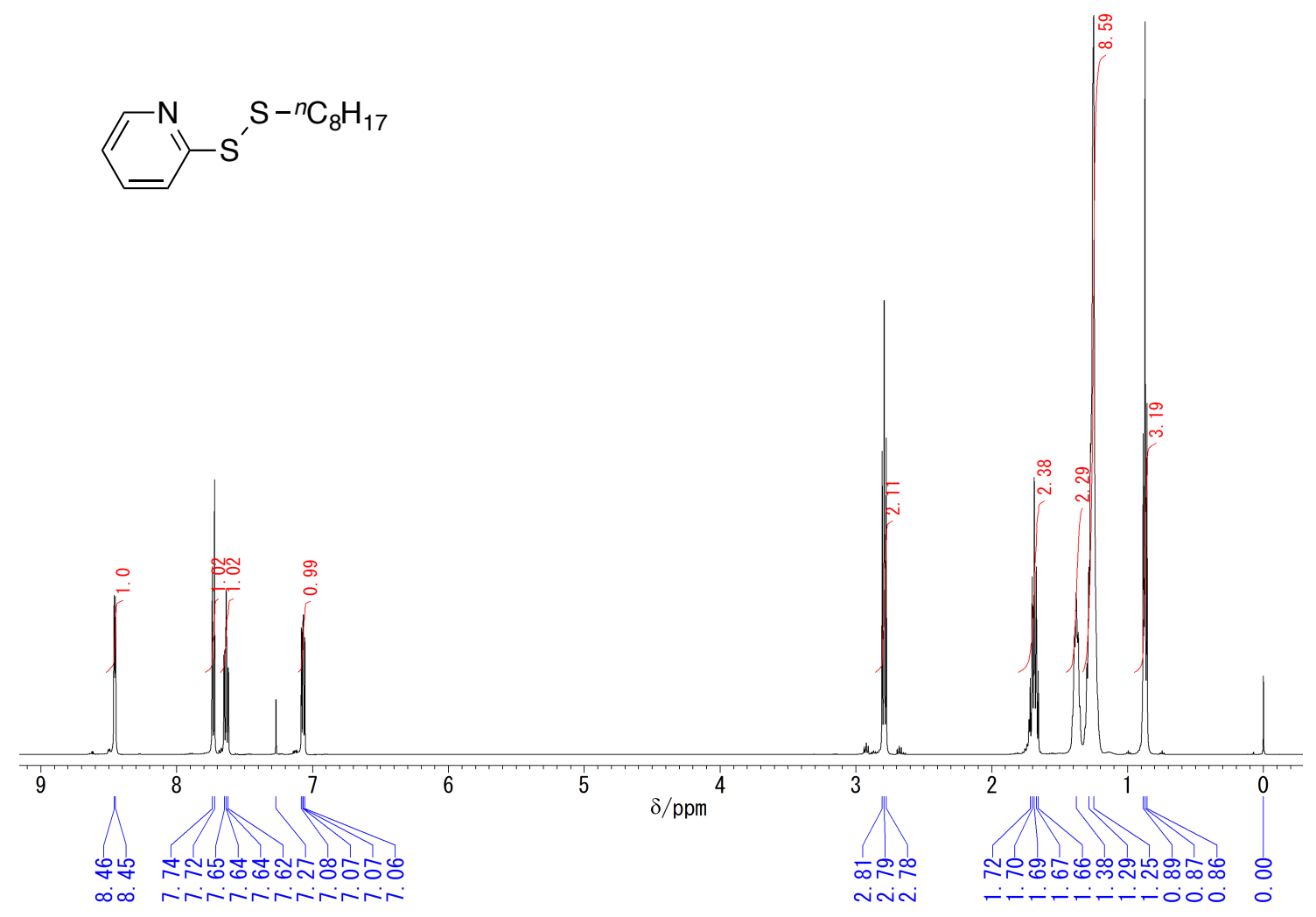

Spectrum S27. 'H NMR $\left(\mathrm{CDCl}_{3}, 500 \mathrm{MHz}\right)$ spectrum of compound 2al.<smiles>COC(=O)CCSSCC(C)(C)C</smiles>
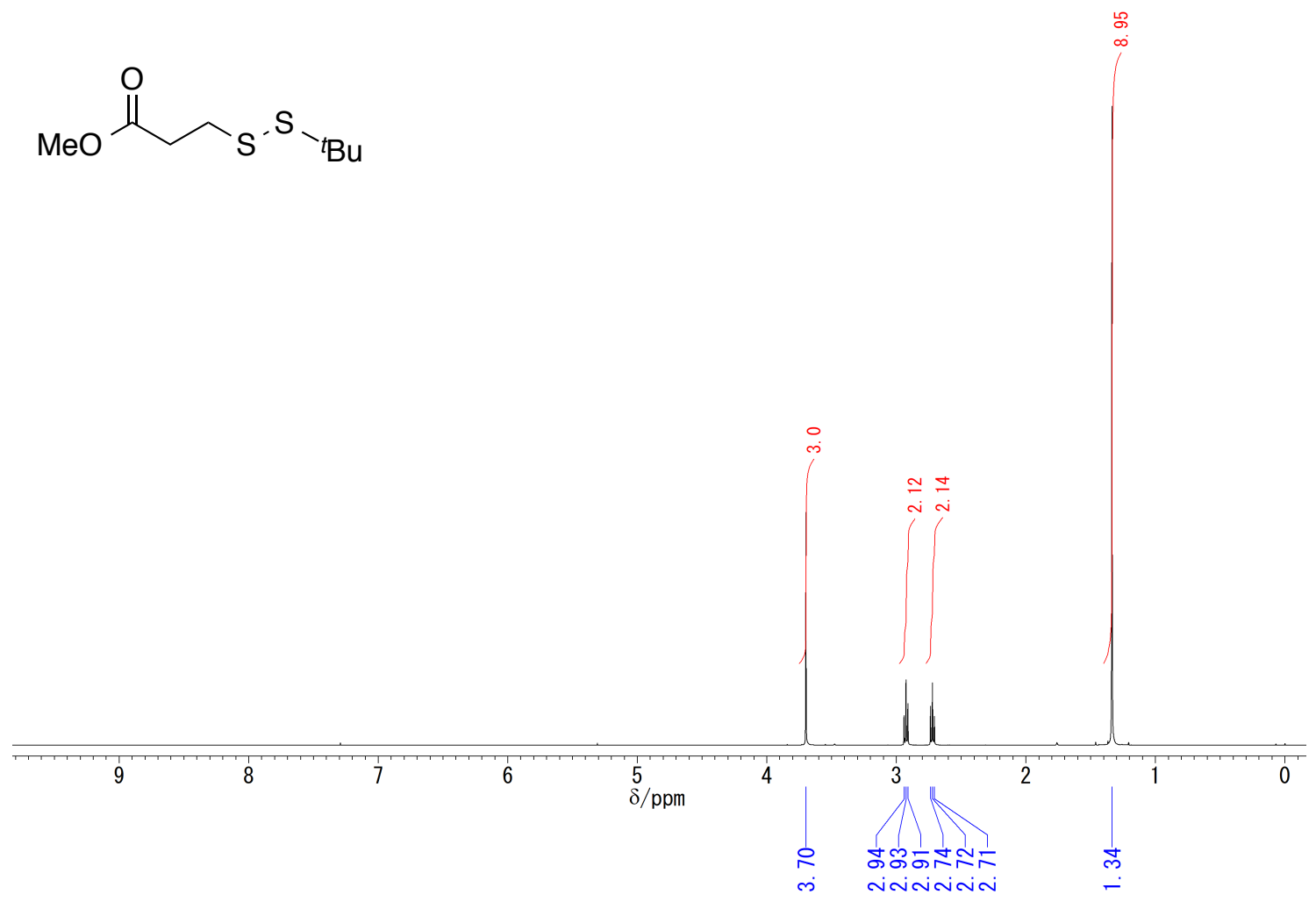

Spectrum S28. 'H NMR $\left(\mathrm{CDCl}_{3}, 500 \mathrm{MHz}\right)$ spectrum of compound 2 oc. 


\section{References}

(S1) a) H. Grande, C. G. Van Schagen, T. Jarbandhan and F. Müller, Helv. Chim. Acta, 1977, 60, 348-366. b) C. G. Van Schagen and F. Müller, Helv. Chim. Acta, 1980, $63,2187-2201$.

(S2) F. Müller, B. M. Donald and D. W. Lemuel, in Methods Enzymol., 1971, 18, 453-458.

(S3) P. Huang, P. Wang, S. Tang, Z. Fu and A. Lei, Angew. Chem., Int. Ed. 2018, 57, 8115-8119.

(S4) L. Song, W. Li, W. Duan, J. An, S. Tang, L. Li and G. Yang, Green Chem., 2019, $21,1432-1438$.

(S5) X. Qiu, X. Yang, Y. Zhang, S. Song, N. Jiao, Org. Chem. Front., 2019, 6, 2220-2225. 